\title{
Analisando a \\ Redundância Ativa \\ em sistemas k-de-n:F \\ sob condições de dependência
}

IRAN MARTINS DO CARMO

Tese apresentada

ao

Instituto de Matemática e Estatística

da

Universidade de São Paulo

para

obtenção do grau

de

Doutor em Estatística

Área de Concentração: Estatística

Orientador: Prof. Dr. VAnderlei da Costa Bueno

São Paulo, janeiro de 2006 


\section{Resumo}

Neste trabalho analisamos o problema da alocação ótima de um, ou vários, spares em um sistema $k-d e-n: F$ de componentes dependentes utilizando a redundância ativa. Os tempos de vida dos spares são independentes entre si e independentes dos tempos de vida dos componentes do sistema. Consideramos a modelagem matemática de duas maneiras: primeiro caracterizamos a redundância ativa através de uma transformação no processo de contagem da falha do componente com uma representação semimartingal; segundo caracterizamos a redundância ativa através da transformação do compensador do mesmo processo de contagem. Os resultados são obtidos usando ordenação estocástica e conceitos da Teoria de Total Positividade. 


\section{Abstract}

In this work we analyze the problem of optimal allocation of, either one, or several, spares in a k-out-of-n:F system of dependent components through active redundancy. The spares lifetimes are independents and independent of the system's components lifetimes. We consider the mathematical formulation in two way: firstly we characterize active redundancy through a transformation of the semimartingale representation of the component failure counting process; second we characterize active redundancy through a transformation of the same process compensator. We get the results using stochastic order and concepts of Total Positivity Theory. 


\section{Sumário}

Introdução 1

1 Redundância ativa dispondo de um único spare 4

1.1 Introdução . . . . . . . . . . . . . . . . . . . . 4

1.2 Modelagem matemática ... . . . . . . . . . . 6

1.2.1 Processo de falha dos componentes . . . . . . . . . . . 6

1.2.2 Representação semimartingal do tempo de vida . . . . . . . . 9

1.2.3 Compensador do tempo de vida de um sistema . . . . . . . 13

1.3 Sistema em série . . . . . . . . . . . . . . . . . . . . 17

1.4 Sistemas $k-d e-n: F \ldots \ldots \ldots . \ldots \ldots 21$

2 Redundância ativa dispondo de vários spares 25

2.1 Introdução . . . . . . . . . . . . . . . . . . . . . . . . 25

2.2 Modelagem matemática . . . . . . . . . . . . . . 26

2.3 Sistema em série . . . . . . . . . . . . . . . . . . . . 27

2.4 Sistema $k-d e-n: F \ldots \ldots \ldots . \ldots . \ldots . \ldots 32$

3 Redundância ativa através da transformação do compensador 36

3.1 Introdução . . . . . . . . . . . . . . . . . . . . . 36

3.2 Transformação do compensador . . . . . . . . . . . . . . 38

3.3 Comparação entre redundância ativa e redundância standby minimal 44 
3.4 Sistema $k-$ de $-n: F$ e função $R R_{2} \ldots \ldots \ldots$. . . . . . 46

3.5 Exemplo construtivo onde $2-\exp \left[-A_{i}(t)\right]$ é $R R_{2} \ldots \ldots . . \ldots 51$

3.6 Sistema $k-$ dee $-n: F$ e as medidas $P_{i}$ e $P_{j} \ldots \ldots . . . . .56$

$\begin{array}{ll}\text { A Apêndice } & 60\end{array}$

$\begin{array}{ll}\text { Bibliografia } & 64\end{array}$ 


\section{Introdução}

Um dos objetivos da Teoria da Confiabilidade é otimizar a confiabilidade de um sistema que depende de seus componentes. Uma das maneiras de proceder com essa otimização é realizar operações de redundância entre o componente do sistema e um outro objeto que denominaremos spare. Existem dois tipos de redundância bem conhecidas na literatura, a redundância ativa e a redundância standby. A primeira representada pelo máximo entre os tempos de vida do sistema e do spare, e a segunda é dada pela convolução desses tempos de vida.

O problema é onde alocar o(s) spare(s) a fim de otimizar o tempo de vida do sistema. Este problema é muito importante na Teoria da Confiabilidade, veja por exemplo os trabalhos de Boland et al (1992), Singh e Misra (1994), Meng (1996), Prasad et al (1999), Mi (2004), Bueno (2005), entre outros.

Boland et al (1992) utiliza um único spare para realizar a operação de redundância. Ele analisa o problema para os dois tipos de redundância em sistemas $k$-de-n: $G$ (sistemas que funcionam se pelo menos $k, k \leq n$, de seus $n$ componentes funcionarem), de componentes estocasticamente independentes. Para redundância ativa ele utiliza a ordenação estocástica dos componentes e mostra que a alocação ótima é no componente estocasticamente mais fraco. Com a redundância standby, ele utiliza a ordenação da razão de verossimilhança, e dá condições suficientes sob 
as quais, nos sistemas em série, a alocação ótima é no componente estocasticamente mais fraco, enquanto que nos sistemas em paralelo, é no componente estocasticamente mais forte.

Nas outras referências encontra-se solução para o problema sob diferentes hipóteses, mas todas consideram os componentes do sistema independentes entre si, exceto o trabalho de Bueno (2005a). Neste trabalho ele definiu um tipo particular de redundância standby, chamando-a de redundância standby minimal, que constituise em substituir o componente, quando este vir a falhar, por um outro, em condições identicas às do que falhara, no instante imediatamente anterior a sua falha. Utiliza este conceito e apresenta condições sob as quais, existe um espaço de probabilidade, onde a alocação ótima é no componente mais fraco, para sistemas $k-d e-n: F$ (sistemas que falham quando $k, 1 \leq k \leq n$, ou mais de seus componentes falharem), com tempos de vida dependentes.

Nesta tese analizamos o problema de otimizar a confiabilidade do sistema através da alocação de um spare utilizando a redundância ativa. Consideramos inicialmente um único spare e depois o caso de vários spares, e admitimos nos dois casos que os componentes sejam estocasticamente dependentes. Neste último caso o tempo de vida do spare é identicamente distribuído ao tempo de vida do componente onde se faz a redundância, e os spares tem tempos de vida independentes entre si e independente dos tempos de vida dos componentes.

Na formulação matemática do problema, utilizamos a Teoria de Processos Estocásticos e Martingais, tendo como principais bibliografias os textos de Aven e U. Jensen (1999), Bremaud (1882) e Dellacherie e Meyer (1980). As conclusões foram obtidas em termos de ordem estocástica, a qual é explorada extensivamente em Shaked e Shanthikumar (1994). 
No Capítulo 1 consideramos a redundância ativa através da transformação do processo de contagem, dispondo de um único spare. Primeiro determinamos o resultado para sistemas em série, somente com a hipótese de ordenação estocástica dos componentes do sistema. Em seguida, com o conceito de função $T P_{2}$, e informação após o nível crítico dos componentes, otimizamos a confiabilidade de sistemas $k-d e-n: F$.

O Capítulo 2 segue os moldes do 1, mas agora os spares são i.i.d. (independente e identicamente distribuido) com os componentes onde se faz a redundância. Portanto, consideramos a redundância ativa utilizando vários spares. Nos sistemas em série, utilizamos a hipótese de ordenação $(P-q . c$.$) dos compensadores dos componentes$ do sistema e nos sistemas $k-d e-n: F$ supomos que $\exp \left[-A_{i}(t)\right]$ seja $T P_{2}$, com informação após o nível crítico dos componentes.

No Capítulo 3 analisamos a redundância ativa considerando a transformação dos compensadores, dispondo de vários spares. Na primeira seção comparamos a confiabilidade dos sistemas utilizando a propriedade de funções reversas de ordem dois $\left(R R_{2}\right)$ e o Teorema de Kwieciński e Szekli (1991). Em seguida obtivemos um exemplo construtivo em que $2-\exp \left[-A_{i}(t)\right]$ é $R R_{2}$, onde $A_{i}(t)$ é o compensador correspondente ao componente com tempo de vida $S_{i}, i=1, \ldots, n$. Na última Seção utilizamos as medidas de probabilidade $P_{i}$ e $P_{j}$, determinadas pela derivada de Radon Nikodym, correspondentes à redundância ativa dos componentes $S_{i}$ e $S_{j}$ respectivamente. 


\section{Capítulo 1}

\section{Redundância ativa dispondo de um único spare}

\subsection{Introdução}

Neste Capítulo consideramos o problema da alocação ótima de um spare para efetuar uma redundância ativa, em um componente, de um sistemas $k-d e-n: F$. Admitimos que os tempos de vida dos componentes do sistema são dependentes, e o tempo de vida do spare é independente dos tempos de vida dos componentes.

Na formulação matemática do problema utilizamos a representação semimartingal para os processos de contagem dos componentes e do sistema, bem como do spare, com seus correspondentes processos intensidade de falha, relativo à informação da dinâmica dos componentes e do spare no tempo. A redundância ativa foi caracterizada a partir da transformação do processo de contagem do componente.

Trabalhamos inicialmente com os sistemas em série, com a hipótese de ordenação estocástica de seus componentes, e em seguida com os sistemas $k-d e-n: F$, utilizando o conceito de função $T P_{2}$ e a informação após o nível crítico dos componentes. A conclusão foi obtida a partir da comparação das esperanças matemática dos com- 
pensadores do sistema, para as $n$ escolhas possíveis. 


\subsection{Modelagem matemática}

\subsubsection{Processo de falha dos componentes}

Consideremos um sistema coerente $\phi$ com $n$ componentes $C_{1}, C_{2}, \ldots C_{n}$ e o vetor aleatório $\mathrm{S}=\left(S_{1}, S_{2}, \ldots, S_{n}\right)$, onde os $S_{i}$ 's, $i=1, \ldots, n$, são variáveis aleatórias positivas, finitas, definidas em um mesmo espaço de probabilidade completo $(\Omega, \Im, P)$, que representam os tempos de vida dos componentes. Assumimos que $P\left(S_{i} \neq S_{j}\right)=1$, para todo $i \neq j, i, j$ em $E=\{1,2, \ldots, n\}$, isto é, os tempos de vida podem ser dependentes mas não admitimos falhas simultâneas. Consideremos também um componente $C$, cujo tempo de vida é indicado pela variável aleatória $S$, positiva, finita, definida em $(\Omega, \Im, P)$, e independente dos $S_{i}$ 's, com $P\left(S_{i} \neq S\right)=1$, para todo $i$ em $E=\{1,2, \ldots, n\}$.

A evolução dos componentes no tempo define um processo pontual marcado, através dos tempos de falha e das correspondentes marcas.

Denotaremos por

$$
S_{(1)}<S_{(2)}<\ldots<S_{(n)}
$$

a ordenação dos tempos de vida $S_{1}, S_{2}, \ldots, S_{n}$, conforme suas ocorrências no tempo, e por

$$
V_{i}=\left\{j: S_{(i)}=S_{j}\right\}
$$

as correspondentes marcas.

Convenciona-se que

$$
S_{(n+1)}=S_{(n+2)}=\ldots=\infty
$$

e

$$
V_{n+1}=V_{n+2}=\ldots=\emptyset
$$


e temos a definição:

\section{Definição 1.1:}

O processo de falha dos componentes é o processo pontual marcado

$$
\left(S_{(m)}, V_{m}\right)_{m \geq 1}
$$

A formulação matemática das observações é dada pela família de sub $\sigma$-álgebras de $\Im$, denotada por

$$
\mathbb{F}=\left(\Im_{t}\right)_{t \geq 0}
$$

onde

$$
\Im_{t}=\sigma\left\{1_{\{S>s\}}, 1_{\left\{S_{(i)}>s, X_{i}=j\right\}}, 0<s \leq t, i, j \in E\right\},
$$

com $1_{\{A\}}$ representando a função indicadora do conjunto A.

A $\sigma$-álgebra $\Im_{t}$ satisfaz as condições usuais de Dellacherie:

- são contínuas à direita, isto é,

$$
\Im_{t}=\Im_{t^{+}}=\bigcap_{s<t} \Im_{s}
$$

- completas (contém todos conjuntos de medida nula).

Intuitivamente, a cada instante $t$ o observador sabe se o(s) evento(s) $\left\{S_{(i)} \leq\right.$ $\left.t, X_{i}=j\right\},\{S \leq t\}$ ocorreu(ram) ou não, e se ele(s) ocorreu(ram), sabe exatamente o(s) valor $(\mathrm{es})$ de $S_{(i)},(S)$ e a marca $X_{i}$.

Seja $\tau$, uma variável aleatória positiva, finita, definida em $(\Omega, \Im, P)$, representando o tempo de vida do sistema $\phi$. O tempo de vida $\tau$ é completamente determinado pelos tempos de vida de seus componentes através da estrutura série-paralelo

$$
\tau=\tau(\mathbf{S})=\min _{1 \leq j \leq r} \max _{i \in K_{j}} S_{i}
$$


(Barlow e Proschan (1981)), onde $K_{j}, 1 \leq j \leq r$, são os conjuntos de cortes minimais de $\phi$, isto é, conjuntos com quantidade mínima de componentes para os quais a falha de todos seus componentes acarreta a falha do sistema.

De maneira equivalente, podemos representar o sistema coerente através de uma função binária de variáveis binárias $\Phi:\{0,1\}^{n} \rightarrow\{0,1\}$, denominada função estrutura do sistema, na forma

$$
\Phi(\mathrm{X}(t))=\min _{1 \leq j \leq r} \max _{i \in K_{j}} X_{i}(t)
$$

onde $K_{j}, 1 \leq j \leq r$, são os conjuntos de cortes minimais, $X_{t}(i)=1_{\left\{S_{i}>t\right\}}, 1 \leq i \leq n$, são processos contínuos à direita, com limite à esquerda, indicando o funcionamento do componente $i$ no instante $t$. O tempo de vida do sistema é

$$
\tau=\inf \left\{t \in \mathbb{R}_{+}: \Phi_{t}=0\right\}
$$

Sejam

$$
N(t)=1_{\{\tau \leq t\}}
$$

e

$$
N_{j}(t)=1_{\left\{S_{j} \leq t\right\}}, \quad j \in E,
$$

os processos pontuais das falhas do sistema e dos componentes respectivamente.

Note que

$$
1_{\left\{S_{j} \leq t\right\}}=\sum_{i \geq 1} 1_{\left\{S_{(i)} \leq t, X_{i}=j\right\}}
$$

e que $N(t)$ e $N_{j}(t)$ são $\mathbb{F}$-submartingais. 


\subsubsection{Representação semimartingal do tempo de vida}

No que segue usaremos os conceitos:

- uma variável aleatória $\tau$, com valores em $R_{+} \cup\{\infty\}$ é chamada um $\mathbb{F}$-tempo de parada se, e somente se, $\{\tau \leq t\} \in \Im_{t}$ para todo $t \geq 0$;

- um $\mathbb{F}$-tempo de parada $\tau$ é chamado previsível se existe uma sequência crescente $\left(\tau_{n}\right)_{n \geq 0}$, de $\mathbb{F}$-tempos de parada, $\tau_{n}<\tau$, tal que $\lim _{n \rightarrow \infty} \tau_{n}=\tau$;

- um $\mathbb{F}$-tempo de parada $\tau$ é totalmente inacessível se $P(\tau=\sigma<\infty)=0$ para todo $\mathbb{F}$-tempo de parada previsível $\sigma$.

Para um estudo de processo estocástico, aplicado à teoria de confiabilidade, recomendamos o livro recente de Aven e Jensen (1999).

Por simplicidade de notação, as relações $C,=, \leq,<e \neq$ entre conjuntos mensuráveis e variáveis aleatórias, respectivamente, serão consideradas válidas com probabilidade um, ou seja, suprimiremos o termo $P$ - q.c., desde que esteja claro no contexto.

O processo

$$
1_{\left\{S_{j} \leq t\right\}}=\sum_{i \geq 1} 1_{\left\{S_{(i)} \leq t, X_{i}=j\right\}}
$$

$j \in E$ é um $\mathbb{F}$-submartingal e pela decomposição de Doob e Meyer (Teorema A.1), temos

$$
1_{\left\{S_{j} \leq t\right\}}=A_{j}(t)+M_{j}(t)
$$

onde $A_{j}(t)$ é um $\mathbb{F}$-processo compensador, e $M_{j}(t)$ um $\mathbb{F}$-martingal. O compensador é um processo crescente, previsível, contínuo á direita, nulo na origem, isto é, $A_{j}(0)=$ 0 , e $A_{j}(t)$ é $P-q . c$ único. O martingal $M_{j}(t)$ é uniformemente integrável (U.I.) e tem média zero. 
Assumiremos que $S_{j}, \quad 1 \leq j \leq n$ e $S$ são $\mathbb{F}$-tempos de parada totalmente inacessíveis. Segue do Lema A.3 que $1_{\left\{S_{j} \leq t\right\}}$ admite a representação semimartingal

$$
1_{\left\{S_{j} \leq t\right\}}=\int_{0}^{t} 1_{\left\{S_{j}>s\right\}} a_{j}(s) d s+M_{j}(t),
$$

onde $a_{j}=\left(a_{j}(t)\right)_{t \geq 0}$ é um processo estocástico progressivamente mensurável, com

$$
E\left[\int_{0}^{t}\left|a_{j}(s)\right| d s\right]<\infty
$$

para todo $t \geq 0$ e $M_{j}=\left(M_{j}(t)\right)_{t \geq 0}$ é um $\mathbb{F}$-martingal U.I. de média zero. $a_{j}$ é chamado o $\mathbb{F}$-processo intensidade de $1_{\left\{S_{j} \leq t\right\}}$ e $A_{j}=\left(A_{j}(t)\right)_{t \geq 0}$, com

$$
A_{j}(t)=\int_{0}^{t} 1_{\left\{S_{j}>s\right\}} a_{j}(s) d s
$$

seu $\mathbb{F}$-processo compensador que é crescente, previsível, contínuo e nulo na origem.

Analogamente, para o processo de contagem $1_{\{S \leq t\}}$, temos

$$
1_{\{S \leq t\}}=\int_{0}^{t} 1_{\{S>s\}} b(s) d s+M(t)
$$

onde $b=(b(t))_{t \geq 0}$ é um processo estocástico progressivamente mensurável com

$$
E\left[\int_{0}^{t}|b(s)| d s\right]<\infty
$$

para todo $t \geq 0$ e $M=(M(t))_{t \geq 0}$ é um $\mathbb{F}$-martingal, U.I., de média zero. $B=$ $(B(t))_{t \geq 0} \mathrm{com}$

$$
B(t)=\int_{0}^{t} 1_{\{S>s\}} b(s) d s,
$$

é seu $\mathbb{F}$-processo compensador e $b$ seu $\mathbb{F}$-processo intensidade.

Definição 1.2: (Redundância Ativa)

Considere $X$ e $Y$ variáveis aleatórias definidas em $(\Omega, \Im, P)$, onde $X$ representa o tempo de vida de um objeto (componente ou sistema) e $Y$ o tempo de vida do spare. A redundância ativa entre o objeto e o spare é a operação que produz o tempo de vida $R$, dado por

$$
R=\max \{X, Y\}=X \vee Y .
$$


Intuitivamente a redundância ativa do componente (ou sistema) equivale a colocar o spare em paralelo com este componente (ou sistema).

Seja $S_{j}^{a}$ o tempo de vida correspondendo à redundância ativa entre os componentes $C_{j}$, com tempo de vida $S_{j}$ e o spare com tempo de vida $S$; o processo pontual associado é representado por $1_{\left\{S_{J}^{a} \leq t\right\}}$. Considerando as representações semimartingais de $1\left\{S_{j} \leq t\right\}$ e $1\{S \leq t\}$, dadas em 1.3 e 1.4 respectivamente, segue o

\section{Teorema 1.3:}

Sejam $S_{j}$ e $S \mathbb{F}$-tempos de parada totalmente inacessíveis, então $1_{\left\{S_{J}^{a} \leq t\right\}}$ é um $\mathbb{F}$ semimartingal regular com representação

$$
1_{\left\{S_{J}^{a} \leq t\right\}}=\int_{0}^{t}\left[1_{\{S \leq s\}} a_{j}(s)+1_{\left\{S_{j} \leq s\right\}} b(s)\right] 1_{\left\{S_{j} \vee S>s\right\}} d s+R(t) .
$$

Prova

Como

$$
1_{\left\{S_{J}^{a} \leq t\right\}}=1_{\left\{S_{j} \vee S \leq t\right\}}=1_{\left\{S_{j} \leq t\right\}} \cdot 1_{\{S \leq t\}},
$$

aplicando a Proposição A.2

$$
\begin{aligned}
1_{\left\{S_{j} \leq t\right\}} \cdot 1_{\{S \leq t\}}= & 1_{\left\{S_{j} \leq 0\right\}} \cdot 1_{\{S \leq 0\}}+\int_{0}^{t} 1_{\left\{S \leq s^{-}\right\}} d\left[1_{\left\{S_{j} \leq s\right\}}\right] \\
& +\int_{0}^{t} 1_{\left\{S_{j} \leq s^{-}\right\}} d\left[1_{\{S \leq s\}}\right]+\sum_{0<s \leq t} \Delta 1_{\left\{S_{j} \leq t\right\}} \Delta 1_{\{S \leq t\}} .
\end{aligned}
$$

Desde que $P\left(S_{j}=S\right)=0$ temos $\sum_{0<s \leq t} \Delta 1_{\left\{S_{j} \leq t\right\}} \Delta 1_{\{S \leq t\}}=0$. 
Assim

$$
\begin{aligned}
1_{\left\{S_{J}^{a} \leq t\right\}}= & \int_{0}^{t} 1_{\{S<s\}} d\left[1_{\left\{S_{j}<s\right\}}\right]+\int_{0}^{t} 1_{\left\{S_{j} \leq s\right\}} d\left[1_{\{S \leq s\}}\right] \\
= & \int_{S}^{t} d\left[1\left\{S_{j} \leq s\right\}\right]+\int_{S_{j}}^{t} d[1\{S \leq s\}] \\
= & 1_{\left\{S_{j} \leq t\right\}}-1_{\left\{S_{j} \leq t \wedge S\right\}}+1_{\{S \leq t\}}-1_{\left\{S \leq t \wedge S_{j}\right\}} \\
= & \int_{0}^{t} 1_{\left\{S_{j}>s\right\}} a_{j}(s) d s+M_{j}(t)-\int_{0}^{t} 1_{\{S>s\}} \cdot 1_{\left\{S_{j}>s\right\}} a_{j}(s) d s-M_{j}(t \wedge S) \\
& +\int_{0}^{t} 1_{\{S>s\}} b(s) d s+M_{S}(t)-\int_{0}^{t} 1_{\left\{S_{j}>s\right\}} \cdot 1_{\{S>s\}} b(s) d s-M_{S}\left(t \wedge S_{j}\right) \\
= & \int_{0}^{t}\left[1_{\{S \leq s\}} a_{j}(s)+1_{\left\{S_{j} \leq s\right\}} b(s)\right] 1_{\left\{S_{j} \vee S>s\right\}} d s+R(t)
\end{aligned}
$$

Portanto $a_{j}^{a}=\left(a_{j}^{a}(t)\right)_{t \geq 0}$ com

$$
a_{j}^{a}(t)=\left[1_{\{S \leq s\}} \cdot a_{j}(s)+1_{\left\{S_{j} \leq s\right\}} \cdot b(s)\right] \cdot 1_{\left\{S_{j}^{a}>s\right\}}
$$

é a $\mathbb{F}$-intensidade de $1_{\left\{S_{J}^{a} \leq t\right\}}$ e $A_{j}^{a}=\left(A_{j}^{a}(t)\right)_{t \geq 0} \operatorname{com} A_{j}^{a}(t)=\int_{0}^{t} a_{j}^{a}(s) d s$, seu $\mathbb{F}$ compensador, enquanto que

$$
R(t)=M_{j}(t)-M_{j}(t \wedge S)+M_{S}(t)-M_{S}\left(t \wedge S_{j}\right)
$$

é um $\mathbb{F}$-martingal,U.I., de média zero. 


\subsubsection{Compensador do tempo de vida de um sistema}

O processo pontual da falha do sistema $\phi, 1_{\{\tau \leq t\}}$, é um $\mathbb{F}$-submartingal, e pela decomposição de Doob e Meyer (Teorema A.1):

$$
1_{\{\tau \leq t\}}=A(t)+M(t)
$$

onde $A(t)$ e $M(t)$ são os $\mathbb{F}$-processos compensador e martingal, respectivamente, de $1_{\{\tau \leq t\}}$.

Uma pergunta natural é qual a relação entre os tempos de vida dos componentes e o tempo de vida do sistema, do ponto de vista dos correspondentes processos de falha? Qual a contribuição do risco de falha dos componentes para predizer o risco de falha do sistema? Arjas (1981) responde essa questão, determinando uma relação entre os $\mathbb{F}$-processos compensadores dos componentes e o $\mathbb{F}$-processo compensador do sistema utilizando o conceito de nível crítico.

Um componente $i$, é crítico para o sistema (Barlow e Proschan (1981)) no instante $t$ se

$$
\Phi\left(1_{i}, \mathbf{X}(t)\right)-\Phi\left(0_{i}, \mathbf{X}(t)\right)=1
$$

onde $\left(1_{i}, \mathrm{x}\right)$ representa o vetor $\mathrm{x}$ com a i-ésima componente fixada e igual a 1 , e $\left(0_{i}, \mathrm{x}\right)$ representa o vetor $\mathrm{x}$ com a i-ésima componente fixada e igual a 0.

No que segue utilizaremos o conceito de nível crítico dos componentes:

Definição 1.4: (Nível crítico)

O nível crítico do componente $i, 1 \leq i \leq n$, é definido por

$$
Y_{i}=\inf \left\{t \geq 0: \Phi\left(1_{i}, \mathbf{X}(t)\right)-\Phi\left(0_{i}, \mathbf{X}(t)\right)=1\right\}
$$


onde

$$
\left(\cdot_{i}, \mathrm{X}(t)\right)=\left(X_{1}(t), \cdots, X_{i-1}(t), \cdot_{i}, X_{i+1}(t), \cdots, X_{n}(t)\right)
$$

Claramente o componente $i$ contribui para a falha do sistema após seu nível crítico, $Y_{i}$, isto é, o primeiro instante a partir do qual sua falha acarreta a falha do sistema.

Observe que o nível crítico de qualquer dos componentes de um sistema em série é $Y_{i}=0$, enquanto que o nível crítico de um componente colocado em paralelo com um subsistema, formando um sistema, é o tempo de vida do subsistema.

Teorema 1.5: (Arjas (1981))

Sob a notação acima, o $\mathbb{F}$-processo compensador do processo $N(t)=1_{\{\tau \leq t\}}$ é

$$
A(t)=\sum_{j=1}^{n}\left[A_{i}(t \wedge \tau)-A_{i}\left(Y_{i}\right)\right]^{+},
$$

onde $A_{i}(t)=\int_{0}^{t} 1_{\left\{S_{i}>s\right\}} a_{s}(i) d s$ e $[a]^{+}=\max \{a, 0\}$.

Definição 1.6: (Sistema $k-d e-n: F)$

Um sistema com $n$ componentes é denominado $k-d e-n: F, 1 \leq k \leq n$, se sua falha ocorre no instante da $k$-ésima falha dos componentes.

O tempo de vida de um sistema $k-d e-n: F$ é a $k$-ésima estatística de ordem $\left(\tau=S_{(k)}\right)$ e os níveis críticos de seus componentes são iguais a $(k-1)$-ésima falha. Portanto $Y_{i}=S_{(k-1)}, i \in E$. Segue como um corolário do Teorema 1.5:

\section{Corolário 1.7:}

Para um sistema $k$-de $-n: F$, com tempo de vida $\tau$, o $\mathbb{F}$-processo compensador de $I_{\{\tau \leq t\}}$ é

$$
A(t)=\sum_{j=1}^{n}\left[A_{i}(t \wedge \tau)-A_{i}(Y)\right]^{+},
$$


onde os $A_{j}(t), j \in E$, são os $\mathbb{F}$-processos compensadores dos processos $1_{\left\{S_{j} \leq t\right\}}$ e $Y$ o nível crítico dos componentes.

Como

$$
\{\tau>t\}=\left\{\cap_{1 \leq j \leq r} \cup_{i \in K_{j}}\left\{S_{i}>t\right\}\right\}
$$

e os $S_{i}$ 's são $\mathbb{F}$-tempos de parada totalmente inacessíveis, $\tau$ é um $\mathbb{F}$-tempo de parada totalmente inacessível, e segue do Teorema A.1 e Lema A.3 que o processo $N(t)=$ $1_{\{\tau \leq t\}}$ tem a seguinte representação semimartingal:

$$
N(t)=\int_{0}^{t} 1_{\tau>s} a(s) d s+M(t)
$$

onde $a=(a(t))_{t \geq 0}$ é um processo estocástico progressivamente mensurável, com

$$
E\left[\int_{0}^{t}|a(s)| d s\right]<\infty
$$

para todo $t \geq 0$ e $M=(M(t))_{t \geq 0}$ é um $\mathbb{F}$-martingal, U.I., de média nula. a é chamado o $\mathbb{F}$-processo intensidade de $N(t)$ e $A=(A(t))_{t \geq 0}$, com

$$
A(t)=\int_{0}^{t} 1_{\tau>s} a(s) d s
$$

seu $\mathbb{F}$-processo compensador, o qual é único, crescente, contínuo, previsível com $A(0)=0$.

\section{Observação 1.8:}

i) Como a confiabilidade do sistema no instante $t$ é $P(\tau>t)=1-P(\tau \leq t)$, e

$$
\begin{aligned}
P(\tau \leq t) & =E[N(t)] \\
& =E[A(t)] \\
& =E\left[\sum_{j=1}^{n}\left[A_{i}(t \wedge \tau)-A_{i}\left(Y_{i}\right)\right]^{+}\right] \\
& =\sum_{j=1}^{n} E\left[\left[A_{i}(t \wedge \tau)-A_{i}\left(Y_{i}\right)\right]^{+}\right]
\end{aligned}
$$


podemos aumentar a confiabilidade do sistema no instante $t, P(\tau>t)$, minimizando a quantidade

$$
E\left[A_{i}(t \wedge \tau)-A_{i}\left(Y_{i}\right)\right]^{+}
$$

com $i \in E$.

ii) No caso da escolha de um componente para realizar a redundância ativa, qual dos $C_{i}$ 's escolher? Isto é, qual dos

$$
E\left[A_{i}(t \wedge \tau)-A_{i}\left(Y_{i}\right)\right]^{+}
$$

minimizar? 


\subsection{Sistema em série}

Desejamos otimizar a confiabilidade de sistemas $k-d e-n: F$ mediante uma redundância ativa sobre um dos componentes do sistema. Denotaremos o tempo de vida de um sistema $k-d e-n: F$ por $\tau_{k: F}(\mathbf{S})=S_{(k)}$, onde $\mathbf{S}=\left(S_{1}, \ldots, S_{n}\right)$ é o vetor aleatório dos tempos de vida dos componentes e indicaremos por

$$
\tau_{k: F}^{i}=\tau_{k: F}\left(S_{1}^{*}, \ldots, S_{i-1}, S_{i}^{a}, S_{i+1}, \ldots, S_{n}\right)
$$

o tempo de vida do sistema com $S_{i}^{a}=S_{i} \vee S$ que é o tempo de vida correspondente à redundância no componente $i$.

Contamos a falha desse sistema através do processo pontual

$$
N^{i}(t)=1_{\left\{\tau_{k: F}^{i} \leq t\right\}}
$$

com o correspondente $\mathbb{F}$-processo compensador, $A^{i}(t), \quad 1 \leq i \leq n$.

Provaremos inicialmente o resultado para sistemas em série, isto é, sistemas $1-d e-n: F$.

\section{Teorema 1.9:}

Sejam $S_{1}, S_{2}, \ldots, S_{n}, \mathbb{F}$-tempos de parada totalmente inacessíveis, representando os tempos de vida dos componentes de um sistema em série; e $S_{i}^{a}, i \in E$, os correspondentes tempos de vida resultantes de uma redundância ativa no componente $i$, onde o spare tem tempo de vida $S$, totalmente inacessível e independente dos $S_{i}$ 's. Se

$$
S_{1} \leq^{s t} S_{2} \leq^{s t} \ldots \leq^{s t} S_{n}
$$

então

$$
\tau_{1: F}^{1} \geq^{s t} \tau_{1: F}^{2} \geq^{s t} \ldots \geq^{s t} \tau_{1: F}^{n} .
$$




\section{Prova}

Em um sistema em série o nível crítico de todos os componentes é 0. Logo, pelo Corolário 1.7, scu $\mathbb{F}$-processo compensador é

$$
A(t)=A\left(t \wedge \tau_{1: F}\right)=\sum_{i=1}^{n} A_{i}\left(t \wedge \tau_{1: F}\right)
$$

onde, de 1.3,

$$
A_{i}(t)=\int_{0}^{t} 1_{\left\{S_{i}>s\right\}} a_{i}(s) d s
$$

Assim, o $\mathbb{F}$-processo compensador do processo $N^{i}(t)$ será

$$
A^{i}(t)=A_{i}^{a}\left(t \wedge \tau_{1: n}^{i}\right)+\sum_{m=1, m \neq i}^{n} A_{m}\left(t \wedge \tau_{1: n}^{i}\right),
$$

com $i \in E$.

Basta provar que $P\left(\tau_{1: F}^{i} \leq t\right) \leq P\left(\tau_{1: F}^{j} \leq t\right)$, , isto é, que

$$
E\left[A^{i}(t)\right] \leq E\left[A^{j}(t)\right]
$$

para $i \leq j, \quad i, j \in E$.

Sem perda de generalidade, é suficiente provar para $n=2$. Se $S$ é o tempo de vida do spare com o $\mathbb{F}$-processo compensador

$$
B(t)=\int_{0}^{t} 1_{\{S>s\}} b(s) d s
$$

segue do Teorema 1.3 que $A^{1}(t)$, o $\mathbb{F}$-processo compensador de $1_{\left\{\tau_{1: F}^{1} \leq t\right\}}$, com $\tau_{1: F}^{1}=$ 
$\tau_{1: F}\left(S_{1}^{a}, S_{2}\right)$, é

$$
\begin{aligned}
A^{1}(t)= & \int_{0}^{t} 1_{\left\{\left(S_{1} \vee S\right) \wedge S_{2}>s\right\}} 1_{\left\{S_{1} \leq s\right\}} 1_{\{S>s\}} b(s) d s \\
& +\int_{0}^{t} 1_{\left\{\left(S_{1} \vee S\right) \wedge S_{2}>s\right\}} 1_{\{S \leq s\}} 1_{\left\{S_{1}>s\right\}} a_{1}(s) d s \\
& +\int_{0}^{t} 1_{\left\{\left(S_{1} \vee S\right) \wedge S_{2}>s\right\}} 1_{\left\{S_{2}>s\right\}} a_{2}(s) d s \\
= & \int_{0}^{t} 1_{\left\{S_{2}>s\right\}} 1_{\left\{S_{1} \leq s\right\}} 1_{\{S>s\}} b(s) d s \\
& +\int_{0}^{t} 1_{\left\{S_{2}>s\right\}} 1_{\{S \leq s\}} 1_{\left\{S_{1}>s\right\}} a_{1}(s) d s \\
& +\int_{0}^{t} 1_{\left\{\left(S_{1} \vee S\right)>s\right\}} 1_{\left\{S_{2}>s\right\}} a_{2}(s) d s .
\end{aligned}
$$

Por outro lado, o $\mathbb{F}$-processo compensador $A^{2}(t)$ de $1_{\left\{\tau_{1: F}^{2} \leq t\right\}}, \operatorname{com} \tau_{1: F}^{2}=\tau_{1: F}\left(S_{1}, S_{2}^{a}\right)$, é

$$
\begin{aligned}
A^{2}(t)= & \int_{0}^{t} 1_{\left\{S_{2} \vee S>s\right\}} 1_{\left\{S_{1}>s\right\}} a_{1}(s) d s \\
& +\int_{0}^{t} 1_{\left\{S_{1}>s\right\}} 1_{\left\{S_{2} \leq s\right\}} 1_{\{S>s\}} b(s) d s \\
& +\int_{0}^{t} 1_{\left\{S_{1}>s\right\}} 1_{\{S \leq s\}} 1_{\left\{S_{2}>s\right\}} a_{2}(s) d s .
\end{aligned}
$$

Assim temos

$$
\begin{aligned}
A^{1}(t)-A^{2}(t)= & \int_{0}^{t} 1_{\{S>s\}} 1_{\left\{S_{2}>s\right\}} a_{2}(s) d s \\
& -\int_{0}^{t} 1_{\{S>s\}} 1_{\left\{S_{1}>s\right\}} a_{1}(s) d s \\
& +\int_{0}^{t} 1_{\{S>s\}}\left[1_{\left\{S_{2}>s\right\}}-1_{\left\{S_{1}>s\right\}}\right] b(s) d s \\
= & \int_{0}^{t} 1_{\{S>s\}}\left[1_{\left\{S_{2}>s\right\}}\left(a_{2}(s)+b(s)\right)-1_{\left\{S_{1}>s\right\}}\left(a_{1}(s)+b(s)\right)\right] d s \\
= & A_{2}\left(t \wedge S \wedge S_{2}\right)+B\left(t \wedge S \wedge S_{2}\right)-A_{1}\left(t \wedge S \wedge S_{1}\right)-B\left(t \wedge S \wedge S_{1}\right) .
\end{aligned}
$$


Mas

$$
\begin{aligned}
E\left[A_{2}\left(t \wedge S \wedge S_{2}\right)+B\left(t \wedge S \wedge S_{2}\right)\right]= & P\left(S_{2} \leq t \wedge S \wedge S_{2}\right)+P\left(S \leq t \wedge S \wedge S_{2}\right) \\
= & 2 P\left(t \wedge S \wedge S_{2} \geq S \wedge S_{2}\right) \\
& -P\left(S \wedge S_{2}<t \wedge S \wedge S_{2} \leq S \vee S_{2}\right) \\
= & 2 P\left(S \wedge S_{2} \leq t\right)-P\left(t \wedge S \wedge S_{2} \geq S \wedge S_{2}\right) \\
= & 2 P\left(S \wedge S_{2} \leq t\right)-P\left(S \wedge S_{2} \leq t\right) \\
= & P\left(S \wedge S_{2} \leq t\right) \\
\leq & P\left(S \wedge S_{1} \leq t\right) \\
= & P\left(S_{1} \leq t \wedge S \wedge S_{1}\right)+P\left(S \leq t \wedge S \wedge S_{1}\right) \\
= & E\left[A_{1}\left(t \wedge S \wedge S_{1}\right)+B\left(t \wedge S \wedge S_{1}\right)\right] .
\end{aligned}
$$

Portanto

$$
E\left[A^{1}(t)\right] \leq E\left[A^{2}(t)\right]
$$

que completa a prova.

Assim, em sistemas em série, concluimos que para otimizar a confiabilidade do sistema deve-se realizar a redundância no componente "mais fraco". 


\subsection{Sistemas $k-d e-n: F$}

Trateremos agora com os sistemas $k-d e-n: F$, e utilizaremos o resultado de Bueno (2005) que propõe analizar o tempo de vida do sistema após o nível crítico de seus componentes, trabalhando com a família de $\sigma$-álgebras $\left(\Im_{Y_{i}+t}\right)_{t \geq 0}$, onde

$$
\Im_{Y_{i}+t}=\left\{A \in \Im_{\infty}: A \cap\left\{Y_{i}+t \leq s\right\} \in \Im_{s}, \quad s \geq 0\right\}
$$

e os tempos de vida

$$
S_{i}^{*}=\left(\left(S_{i}-Y_{i}\right)^{+} \mid \Im_{Y_{i}}\right)
$$

$i \in E$, com o processo de contagem correspondente

$$
1_{\left\{S_{i}^{*} \leq t\right\}}=E\left[N_{i}\left(Y_{i}+t\right)-N_{i}\left(Y_{i}\right) \mid \Im_{Y_{i}}\right]=E\left[1_{\left\{Y_{i}<S_{i} \leq Y_{i}+t\right\}} \mid \Im_{Y_{i}}\right]
$$

Segue que

$$
C_{i}(t)=E\left[A_{i}\left(Y_{i}+t\right)-A_{i}\left(Y_{i}\right) \mid \Im_{Y_{i}}\right]
$$

é o $\Im_{Y_{i}+t}$-processo compensador de $1_{\left\{S_{i}^{*} \leq t\right\}}$. Neste contexto obtém a representação de um sistema coerente como uma estrutura em série (Teorema A.5).

Para obter o próximo teorema, utilizaremos o Teorema A.5 e a definição de funções $T P_{2}$, Totalmente Positiva de Ordem 2 (Total Positivity of Order 2), da Teoria de Positividade Total (Karlin(1968)).

Definição 1.10: (Função $T P_{2}$ )

Uma função real, bivariada, positiva $K(x, y)$, definida para $-\infty<x<\infty$, $-\infty<y<\infty$, é $\left(T P_{2}\right)$ se, e somente se,

$$
K\left(x_{1}, y_{2}\right) K\left(x_{2}, y_{1}\right) \leq K\left(x_{1}, y_{1}\right) K\left(x_{2}, y_{2}\right)
$$

para todo $-\infty<x_{1}<x_{2}<\infty, \quad-\infty<y_{1}<y_{2}<\infty$. 


\section{Teorema 1.11:}

Sejam $S_{1}, S_{2}, \ldots, S_{n} \mathbb{F}$-tempos de parada totalmente inacessíveis, representando os tempos de vida de $n$ componentes de um sistema $k-d c-n: F$; e $S_{i}^{a}, i \in E$, os correspondentes tempos de vida resultantes de uma redundância ativa onde o spare tem tempo de vida $S$, totalmente inacessível e independente dos $S_{i}$ 's. Considere $S_{i}^{* \prime s}, i \in E$, como em 1.7. Sob $\Im_{Y+t}$, se $K(i, t)=\exp \left[-A_{i}(t)\right]$, com $t \in[0, \infty)$, é $T P_{2}$, então

$$
\tau_{k: F}^{1} \geq^{s t} \tau_{k: F}^{2} \geq^{s t} \ldots \geq^{s t} \tau_{k: F}^{n}
$$

\section{Prova}

Do Corolário 1.7, o $\mathbb{F}$-processo compensador do sistema $k-d e-n: F$ é

$$
A(t)=A\left(t \wedge \tau_{k: F}\right)=\sum_{i=1}^{n}\left[A_{i}\left(t \wedge \tau_{k: F}\right)-A_{i}(Y)\right]^{+}
$$

onde $Y=S_{(k-1)}$.

Então pelos Teoremas A.5 e 1.9, basta provar que, para todo $1 \leq i, j \leq n$,com $i<j$,

$$
\begin{aligned}
S_{i}^{*} & =\left(\left(S_{i}-Y\right)^{+} \mid \Im_{Y}\right) \\
& \leq^{s t}\left(\left(S_{j}-Y\right)^{+} \mid \Im_{Y}\right) \\
& =S_{j}^{*} .
\end{aligned}
$$

Da hipótese de que $\exp \left[-A_{j}(t)\right]$ é $T P_{2}$, temos

$$
\exp \left[-A_{i}(Y)\right] \cdot \exp \left[-A_{j}(t+Y)\right] \geq \exp \left[-A_{i}(t+Y)\right] \cdot \exp \left[-A_{j}(Y)\right]
$$

e portanto

$$
A_{j}(t+Y)-A_{j}(Y) \leq A_{i}(t+Y)-A_{i}(Y)
$$


Assim

$$
\begin{aligned}
P\left(S_{j}^{*} \leq t\right) & =E\left[C_{j}(t)\right] \\
& =E\left[A_{j}(t+Y)-A_{j}(Y)\right] \\
& \leq E\left[A_{i}(t+Y)-A_{i}(Y)\right] \\
& =E\left[C_{i}(t)\right] \\
& =P\left(S_{i}^{*} \leq t\right),
\end{aligned}
$$

concluindo a prova.

Se $\exp \left[-A_{i}(t)\right]$ é $T P_{2}$ temos que, para $s \leq t$,

$$
A_{j}(t)-A_{j}(s) \leq A_{i}(t)-A_{i}(s)
$$

Fazendo $s=0$ e lembrando que $A_{i}(0)=0$, para todo $i$, temos $A_{i}(t) \geq A_{j}(t), P$-q.c., para todo $1 \leq i, j \leq n$, com $i<j$. Logo,

$$
\mathbb{E} A_{i}(t) \geq \mathbb{E} A_{j}(t)
$$

e temos

$$
P\left(S_{i} \leq t\right) \geq P\left(S_{j} \leq t\right)
$$

ou seja

$$
S_{i} \leq^{s t} S_{j}
$$

e interpretamos o componente $i$, como sendo "mais fraco" (em termos de desigualdade estocástica) do que o componente $j$.

A ordenação $A_{i} \leq A_{j}$ acima é a Ordem do Risco Acumulado, que implica na Ordem Estocástica Multivariada, enquanto que a Ordem da Razão de Verossimelhança 
Multivariada implica na Ordem do Risco Acumulado. Para mais detalhes veja por exemplo Shaked e Shanthikumar (1994).

\section{Observação 1.12:}

i) Sob o Teorema 1.11, entendemos que, após o nível crítico, otimizaremos a confiabilidade do sistema através da redundância ativa, alocando o componente redundante ao componente mais fraco do sistema $k-d e-n: F$.

ii) Desde que, em nenhum momento, não mencionamos qualquer relação entre os componentes do sistema, nossos resultados são válidos para componentes estocasticamente dependentes.

iii) O Teorema 1.9, generaliza o Teorema de Boland(1992), A.5, para o caso de sistemas em série. 


\section{Capítulo 2}

\section{Redundância ativa dispondo de vários spares}

\subsection{Introdução}

Neste Capítulo a modelagem matemática é semelhante à do Capítulo 1 e a redundância ativa foi caracterizada a partir da transformação do processo de contagem do componente.

A diferença essencial entre este Capítulo e o anterior, é que a redundância é efetuada considerando o tempo de vida do spare independente e identicamente distribuido com o correspondente tempo de vida do componente onde se faz a redundância ativa e independente dos demais componentes. Portanto, dispomos de vários spares, e os componentes do sistema podem ser dependentes.

Trabalhamos com os sistemas em série utilizando a hipótese de ordenação ( $P$ q.c.) entre os compensadores dos componentes do sistema, e nos sistemas $k-d e-$ $n$ : F utilizamos o conceito de função $T P_{2}$ com informação após o nível crítico dos componentes. A conclusão foi obtida a partir da comparação das esperanças matemática dos compensadores do sistema, para as $n$ escolhas possíveis. 


\subsection{Modelagem matemática}

Como na Seção 1.2, considere o vetor $\left(S_{1}, S_{2}, \ldots, S_{n}\right)$ dos tempos de vida de $n$ componentes os quais são variáveis aleatórias positivas e finitas, definidas em um espaço de probabilidade completo $(\Omega, \Im, P)$, com $P\left(S_{i} \neq S_{j}\right)=1$, para todo $i \neq j, i, j$ em $E=\{1,2, \ldots, n\}$.

Sejam $T_{j}, \quad 1 \leq j \leq n$, os tempos de vida de $n$ spares, que são variáveis aleatórias positivas e finitas, definidas no mesmo espaço de probabilidade completo $(\Omega, \Im, P)$, independentes e identicamente distribuídos aos $S_{j}$, com $P\left(S_{i} \neq T_{j}\right)=1$, para todo $i \neq j, i, j$ em $E$.

Neste contexto, a formulação matemática de nossas observações é dado pela família de sub $\sigma$-álgebras de $\Im$, denotada por $\mathbb{F}=\left(\Im_{t}\right)_{t \geq 0}$, onde

$$
\Im_{t}=\sigma\left\{1_{\left\{T_{j}>s\right\}}, 1_{\left\{S_{(i)}>s, X_{i}=j\right\}}, 0<s \leq t, 1 \leq i \leq n, j \in E\right\}
$$

Assumiremos que $S_{i}$, e $T_{j}, i, j \in E$, são $\mathbb{F}$-tempos de parada totalmente inacessíveis.

Como na Seção 1.3, $1_{\left\{S_{j} \leq t\right\}}$ tem a representação semimartingal

$$
1_{\left\{S_{j} \leq t\right\}}=\int_{0}^{t} 1_{\left\{S_{j}>s\right\}} a_{j}(s) d s+M_{j}(t) .
$$

Para o processo de contagem $1_{\left\{T_{j} \leq t\right\}}, 1 \leq j \leq n$, correspondendo aos tempos de vida dos spares, temos

$$
1_{\left\{T_{j} \leq t\right\}}=\int_{0}^{t} 1_{\left\{T_{j}>s\right\}} b_{j}(s) d s+M_{j}^{s}(t),
$$

onde $\left(b_{j}(t)\right)_{t \geq 0}$ é um processo estocástico progressivamente mensurável com

$$
E\left[\int_{0}^{t}\left|b_{j}(s)\right| d s\right]<\infty
$$


para todo $t \geq 0$ e $M_{j}^{s}(t)$ é um $\mathbb{F}$-martingal,U.I., de média zero, com

$$
B_{j}(t)=\int_{0}^{t} 1_{\left\{T_{j}>s\right\}} b_{j}(s) d s
$$

o $\mathbb{F}$-processo compensador correspondente.

O processo de contagem $1_{\left\{S_{j}^{a} \leq t\right\}}$ correspondente à redundância ativa do componente com tempo de vida $S_{j}$ e do spare com tempo de vida $T_{j}$, isto é $S_{j}^{a}=S_{j} \vee T_{j}$, tem representação semimartingal dada pelo Teoremä 1.3

$$
\begin{aligned}
1_{\left\{S_{j}^{a} \leq t\right\}} & =1_{\left\{S_{j} \leq t\right\}} 1_{\left\{T_{j} \leq t\right\}} \\
& =\int_{0}^{t}\left[1_{\left\{T_{j} \leq s\right\}} a_{j}(s)+1_{\left\{S_{j} \leq s\right\}} b_{j}(s)\right] 1_{\left\{S_{j} \vee T_{j}>s\right\}} d s+R(t)
\end{aligned}
$$

onde $(R(t))_{t \geq 0}$ é um $\mathbb{F}$-martingale, U.I., de média zero.

No que segue assumiremos as seguintes hipóteses sobre os tempos de vida $S_{i}$, $1 \leq i \leq n$ e sobre os tempos de vida dos spares $T_{i}, 1 \leq i \leq n$ :

\section{Hipótese A:}

A0) $S_{i}$ 's e $T_{i}{ }^{\prime}$ s, $1 \leq i \leq n$ são totalmente inacessíveis;

A1) $T_{i}$ e $T_{j}, i \neq j, \quad 1 \leq i, j \leq n$, são independentes;

A2) $T_{i}$ e $S_{i}$ são identicamente distribuidos, $1 \leq i \leq n$;

A3) $T_{i}$ é independente de $S_{j}, 1 \leq i, j \leq n$.

\subsection{Sistema em série}

Como no Capítulo 1, provaremos primeiro o resultado para sistemas em série, e em seguida para sistemas $k-d e-n: F$. 


\section{Teorema 2.1:}

Em um sistema em série, sejam $S_{i}$ e $T_{i}, 1 \leq i \leq n$, os tempos de vida dos componentes e dos spares respectivamente, satisfazendo a Hipótese A. Se

$$
A_{1}(t) \geq A_{2}(t) \geq \ldots \geq A_{n}(t)
$$

$P-q . c .$, então

$$
\tau_{1: F}^{1} \leq^{s t} \tau_{1: F}^{2} \leq^{s t} \ldots \leq^{s t} \tau_{1: F}^{n}
$$

\section{Prova}

Como o sistemas é em série, seu $\mathbb{F}$-processo compensador é

$$
A(t)=A\left(t \wedge \tau_{1: F}\right)=\sum_{i=1}^{n} A_{i}\left(t \wedge \tau_{1: F}\right)
$$

com

$$
A_{i}(t)=\int_{0}^{t} 1_{\left\{S_{i}>s\right\}} a_{i}(s) d s
$$

Devemos provar que $E\left[A^{1}(t)\right] \geq E\left[A^{2}(t)\right]$.

Seja $T_{i}$ o tempo de vida do spare com o $\mathbb{F}$-processo compensador

$$
B_{i}(t)=\int_{0}^{t} 1_{\left\{T_{i}>s\right\}} b_{i}(s) d s .
$$


Então, da equação 2.4, o $\mathbb{F}$-processo compensador $A^{1}(t)$ de $1_{\left\{\tau_{1: F}^{1} \leq t\right\}}$, com $\tau_{1: F}^{1}=$ $\tau_{1: F}\left(S_{1}^{a}, S_{2}\right)$, é

$$
\begin{aligned}
A^{1}(t)= & \int_{0}^{t} 1_{\left\{\left(S_{1} \vee T_{1}\right) \wedge S_{2}>s\right\}} 1_{\left\{S_{1} \leq s\right\}} 1_{\left\{T_{1}>s\right\}} b_{1}(s) d s \\
& +\int_{0}^{t} 1_{\left\{\left(S_{1} \vee T_{1}\right) \wedge S_{2}>s\right\}} 1_{\left\{T_{1} \leq s\right\}} 1_{\left\{S_{1}>s\right\}} a_{1}(s) d s \\
& +\int_{0}^{t} 1_{\left\{\left(S_{1} \vee T_{1}\right) \wedge S_{2}>s\right\}} 1_{\left\{S_{2}>s\right\}} a_{2}(s) d s \\
= & \int_{0}^{t} 1_{\left\{S_{2}>s\right\}} 1_{\left\{S_{1} \leq s\right\}} 1_{\left\{T_{1}>s\right\}} b_{1}(s) d s \\
& +\int_{0}^{t} 1_{\left\{S_{2}>s\right\}} 1_{\left\{T_{1} \leq s\right\}} 1_{\left\{S_{1}>s\right\}} a_{1}(s) d s \\
& +\int_{0}^{t} 1_{\left\{\left(S_{1} \vee T_{1}\right)>s\right\}} 1_{\left\{S_{2}>s\right\}} a_{2}(s) d s \\
= & A_{2}\left(t \wedge\left(T_{1} \vee S_{1}\right) \wedge S_{2}\right)+B_{1}\left(t \wedge T_{1} \wedge S_{2}\right) \\
& -B_{1}\left(S_{1}\right)+A_{1}\left(t \wedge S_{1} \wedge S_{2}\right)-A_{1}\left(T_{1}\right) .
\end{aligned}
$$

Análogamente $A^{2}(t)$, o $\mathbb{F}$-processo compensador de $1_{\left\{\tau_{1: F}^{2} \leq t\right\}}, \operatorname{com} \tau_{1: F}^{2}=\tau_{1: F}\left(S_{1}, S_{2} \vee\right.$ $\left.T_{2}\right)$ é

$$
\begin{aligned}
A^{2}(t)= & \int_{0}^{t} 1_{\left\{S_{2} \vee T_{2}>s\right\}} 1_{\left\{S_{1}>s\right\}} a_{1}(s) d s \\
& +\int_{0}^{t} 1_{\left\{S_{1}>s\right\}} 1_{\left\{S_{2} \leq s\right\}} 1_{\left\{T_{2}>s\right\}} b_{2}(s) d s \\
& +\int_{0}^{t} 1_{\left\{S_{1}>s\right\}} 1_{\left\{T_{2} \leq s\right\}} 1_{\left\{S_{2}>s\right\}} a_{2}(s) d s \\
= & {\left[A_{1}\left(t \wedge\left(T_{2} \vee S_{2}\right) \wedge S_{1}\right)+B_{2}\left(t \wedge T_{2} \wedge S_{1}\right)\right.} \\
& \left.-B_{2}\left(S_{2}\right)+A_{2}\left(t \wedge S_{1} \wedge S_{2}\right)-A_{2}\left(T_{2}\right)\right] .
\end{aligned}
$$

Como $E\left[A_{1}\left(T_{1}\right)\right]+E\left[B_{1}\left(S_{1}\right)\right]=1$ e $E\left[A_{2}\left(T_{2}\right)\right]+E\left[B_{2}\left(S_{2}\right)\right]=1$, é suficiente provar que

$$
E\left[A_{2}\left(t \wedge\left(T_{1} \vee S_{1}\right) \wedge S_{2}\right)\right]-E\left[A_{2}\left(t \wedge S_{1} \wedge S_{2}\right)\right]+B_{1}\left(t \wedge T_{1} \wedge S_{2}\right)
$$




$$
\geq E\left[A_{1}\left(t \wedge\left(T_{2} \vee S_{2}\right) \wedge S_{1}\right)\right]-E\left[A_{1}\left(t \wedge S_{1} \wedge S_{2}\right)\right]+B_{2}\left(t \wedge T_{2} \wedge S_{1}\right)
$$

Mas

$$
\begin{aligned}
& E\left[A_{2}\left(t \wedge\left(T_{1} \vee S_{1}\right) \wedge S_{2}\right)\right]-E\left[A_{2}\left(t \wedge S_{1} \wedge S_{2}\right)\right] \\
& =P\left(S_{2} \leq\left(t \wedge T_{1}\right) \vee\left(t \wedge S_{1}\right)\right)-P\left(S_{2} \leq t \wedge S_{1}\right) \\
& =P\left(t \wedge T_{1} \wedge S_{1} \leq S_{2} \leq t \wedge T_{1}\right) \\
& =P\left(S_{1} \leq S_{2} \leq t \wedge T_{1}\right) \\
& =\mathbb{E}\left[1_{\left\{S_{1} \leq S_{2} \leq t \wedge T_{1}\right\}}\right] \\
& =E\left\{E\left[1_{\left\{S_{1} \leq S_{2} \leq t \wedge T_{1}\right\}} \mid \Im_{S_{1}}\right]\right\} \\
& =E\left\{E\left[A_{2}\left(t \wedge T_{1}\right)-A_{2}\left(\left(S_{1}\right) \mid \Im_{S_{1}}\right]\right\}\right. \\
& =E\left[A_{2}\left(t \wedge T_{1}\right)-A_{2}\left(\left(S_{1}\right)\right] .\right.
\end{aligned}
$$

Semelhantemente,

$$
\begin{aligned}
& E\left[A_{1}\left(t \wedge\left(T_{2} \vee S_{2}\right) \wedge S_{1}\right)\right]-E\left[A_{1}\left(t \wedge S_{1} \wedge S_{2}\right)\right] \\
& =P\left(S_{2} \leq S_{1} \leq t \wedge T_{2}\right) \\
& =E\left[A_{1}\left(t \wedge T_{2}\right)-A_{1}\left(\left(S_{2}\right)\right] .\right.
\end{aligned}
$$

Como

$$
\begin{aligned}
E\left[B_{1}\left(t \wedge T_{1} \wedge S_{2}\right)\right] & =\int_{0}^{t} P\left(S_{2} \geq x\right) d F_{T_{1}}(x) \\
& =1-E\left[A_{2}\left(t \wedge T_{1}\right)\right]-P\left(S_{2}>t\right) P\left(S_{1}>t\right)
\end{aligned}
$$

e

$$
\begin{aligned}
E\left[B_{2}\left(t \wedge T_{2} \wedge S_{1}\right)\right] & =\int_{0}^{t} P\left(S_{1} \geq x\right) d F_{T_{2}}(x) \\
& =1-E\left[A_{1}\left(t \wedge T_{2}\right)\right]-P\left(S_{2}>t\right) P\left(S_{1}>t\right),
\end{aligned}
$$


A hipótese do teorema implica que

$$
A_{1}\left(S_{2}\right) \geq A_{2}\left(S_{1}\right)
$$

Portanto, das relações de 2.5 a 2.11 temos que

$$
E\left[A^{1}(t)\right] \geq E\left[A^{2}(t)\right]
$$

o que completa a prova.

Do Teorema 2.1, concluimos que para otimizar a confiabilidade de sistemas em série, dispondo de vários spares, deve-se realizar a redundância no componente "mais forte". 
2.4 Sistema $k-d e-n: F$

Para provar o teorema a seguir, ret.omamos o Teorema da Representação em Série de Bueno (2005b), A.5 e a definição de função $T P_{2}$, da Seção 1.4

\section{Teorema 2.2:}

Em um sistema $k-d e-n: F$, sejam $S_{i}{ }^{\prime}$ s e $T_{i}{ }^{\prime}$ s, $1 \leq i \leq n$, representando os tempos de vida dos componentes e dos spares, respectivamente, satisfazendo a Hipótese A. Sob a $\sigma$-álgebra $\Im_{Y+t}$, se $K(j, t)=\exp \left[-A_{j}(t)\right]$ é $T P_{2}$, então $\tau_{k: F}^{i} \leq^{s t} \tau_{k: F}^{j}$, com $i \leq j, 1 \leq i, j \leq n$ e $t \in[0, \infty)$.

\section{Prova}

O $\mathbb{F}$-processo compensador de um sistema $k-d e-n: F$ com nível crítico $Y=S_{(k-1)}$ é

$$
A(t)=A\left(t \wedge \tau_{k: F}\right)=\sum_{i=1}^{n}\left[A_{i}\left(t \wedge \tau_{k: F}\right)-A_{i}(Y)\right]^{+} .
$$

A prova segue dos Teoremas A.5 e 2.1 , bastando provar que $C_{i}(t) \geq C_{j}(t)$ para todo $i<j, i, j \in E$, onde $C_{i}(t)=E\left[A_{i}(Y+t)-A_{i}\left(Y_{i}\right) \mid \Im_{Y}\right]$ é o $\Im_{Y+t}$-processo compensador de $S_{i}^{*}=\left(\left(S_{i}-Y\right)^{+} \mid \Im_{Y}\right)$.

Como por hipótese, $K(j, t)=\exp \left[-A_{j}(t)\right], \quad 1 \leq j \leq n, \quad t \in[0, \infty)$ é $T P_{2}$, nós temos para $s<t$ que

$$
A_{1}(s)-A_{1}(t) \leq A_{2}(s)-A_{2}(t)
$$

e portanto, para todo $B \in \Im_{Y}$ 


$$
\begin{aligned}
\int_{B} E\left[A_{2}(s+Y)-A_{2}(t+Y) \mid \Im_{Y}\right] d P & =\int_{B}\left(A_{2}(s+Y)-A_{2}(t+Y)\right) d P \\
& \geq \int_{B}\left(A_{1}(s+Y)-A_{1}(t+Y)\right) d P \\
& =\int_{B} E\left[A_{1}(s+Y)-A_{1}(t+Y) \mid \Im_{Y}\right] d P
\end{aligned}
$$

assim

$$
\int_{B}\left\{E\left[A_{2}(s+Y)-A_{2}(t+Y) \mid \Im_{Y}\right]-E\left[A_{1}(s+Y)-A_{1}(t+Y) \mid \Im_{Y}\right]\right\} d P \geq 0 .
$$

Fixando

$$
B_{n}=\left\{E\left[A_{2}(s+Y)-A_{2}(t+Y) \mid \Im_{Y}\right]-E\left[A_{1}(s+Y)-A_{1}(t+Y) \mid \Im_{Y}\right]<-\frac{1}{n}\right\}
$$

como $B_{n} \in \Im_{Y}$, segue que

$\int_{B_{n}}\left\{E\left[A_{2}(s+Y)-A_{2}(t+Y) \mid \Im_{Y}\right]-E\left[A_{1}(s+Y)-A_{1}(t+Y) \mid \Im_{Y}\right]\right\} d P \leq-\frac{1}{n} P\left(B_{n}\right)=0$

e $P\left(B_{n}\right)=0$ para todo $n \geq 1$.

Mas

$$
\begin{aligned}
P\left\{E\left[A_{2}(s+Y)-A_{2}(t+Y) \mid \Im_{Y}\right]\right. & \left.-E\left[A_{1}(s+Y)-A_{1}(t+Y) \mid \Im_{Y}\right]<0\right\} \\
& =P\left(\bigcup_{n=1}^{\infty} B_{n}\right) \\
& \leq \sum_{n=1}^{\infty} P\left(B_{n}\right) \\
& =0
\end{aligned}
$$

e portanto

$$
E\left[A_{2}(s+Y)-A_{2}(t+Y) \mid \Im_{Y}\right] \geq E\left[A_{1}(s+Y)-A_{1}(t+Y) \mid \Im_{Y}\right]
$$


$P-$ q.c., finalizando a prova.

Da última desigualdade acima, podemos escrever

$$
\begin{aligned}
\frac{\exp \left[-E\left[A_{1}(Y+t)-A_{1}(Y) \mid \Im_{Y}\right]\right]}{\exp \left[-E\left[A_{1}(Y+s)-A_{1}(Y) \mid \Im_{Y}\right]\right]} & =\frac{\exp \left[-E\left[A_{1}(Y+t) \mid \Im_{Y}\right]\right]}{\exp \left[-E\left[A_{1}(Y+s) \mid \Im_{Y}\right]\right]} \\
& \leq \frac{\exp \left[-E\left[A_{2}(Y+t) \mid \Im_{Y}\right]\right]}{\exp \left[-E\left[A_{2}(Y+s) \mid \Im_{Y}\right]\right]} \\
& =\frac{\exp \left[-E\left[A_{2}(Y+t)-A_{2}(Y) \mid \Im_{Y}\right]\right]}{\exp \left[-E\left[A_{2}(Y+s)-A_{2}(Y) \mid \Im_{Y}\right]\right]}
\end{aligned}
$$

e podemos enunciar a proposição:

\section{Proposição 2.3:}

Se $\exp \left[-A_{i}(t)\right]$ é $T P_{2}$, então $\exp \left[-C_{i}(t)\right]$ é $T P_{2}$.

\section{Observações 2.4:}

Sob o Teorema acima, isto é, depois do nível crítico, entendemos que é preferível fazer a redundância ativa no componente mais forte (no sentido de ordenação estocástica). Como na seção anterior, não mencionamos qualquer relação entre os componentes, pontanto nossos resultados são válidos para componente estocasticamente dependentes.

A seguir daremos um exemplo de distribuição, derivada de um processo de Weibull, veja Bain (1978), em que $\exp \left[-A_{j}(t)\right]$ tem a propriedade $T P_{2}$.

\section{Exemplo 2.5:}

Sejam $\left\{S_{1}, \ldots, S_{n}\right\}$ os tempos de vida ordenados, com função de sobrevivência condicional dada por:

$$
\bar{F}\left(s_{i} \mid s_{1}, s_{2}, \ldots s_{i-1}\right)=\exp \left[-\left(\frac{s_{i}-\eta_{i}}{\theta}\right)^{\beta}+\left(\frac{s_{i-1}-\eta_{i}}{\theta}\right)^{\beta}\right]
$$


para $\eta_{i} \vee s_{i-1}<s_{i}$, onde $s_{i}$ são as observações ordenadas, tendo função densidade:

$$
\begin{aligned}
f\left(s_{1}, s_{2}, \ldots, s_{n}\right)= & \prod_{i=1}^{n} f\left(s_{i} \mid s_{1}, s_{2}, \ldots s_{i-1}\right) \\
= & \left(\frac{\beta}{\theta}\right)\left(\frac{s_{1}-\eta_{1}}{\theta}\right)^{\beta-1} \exp \left[\left(\frac{s_{1}-\eta_{1}}{\theta}\right)^{\beta}\right] \\
& \cdot \prod_{i=2}^{n}\left(\frac{\beta}{\theta}\right)\left(\frac{s_{i}-\eta_{i}}{\theta}\right)^{\beta-1} \exp \left[-\left(\frac{s_{i}-\eta_{i}}{\theta}\right)^{\beta}+\left(\frac{s_{i-1}-\eta_{i}}{\theta}\right)^{\beta}\right] .
\end{aligned}
$$

Segue que

$$
\begin{aligned}
d A_{i}\left(s \mid s_{1}, s_{2}, \ldots s_{i-1}\right) & =\frac{f\left(s \mid s_{1}, s_{2}, \ldots s_{i-1}\right)}{\bar{F}\left(\mid s_{1}, s_{2}, \ldots s_{i-1}\right)} \\
& =\left(\frac{\beta}{\theta}\right)\left(\frac{s-\eta_{i}}{\theta}\right)^{\beta-1}
\end{aligned}
$$

com $s_{i-1} \leq s<s_{i}, \quad s_{0}=0$

Em particular, se $\beta=2, S_{i}$ tem distribuição $\left(I F R \mid \Im_{s}^{i}\right)$, tal que $\bar{F}\left(s_{i} \mid s_{1}, s_{2}, \ldots s_{i-1}\right)$ é $T P_{2}$ sobre $s_{i}$ e $\eta_{i}$ com

$$
\Im_{t}^{i}=\sigma\left\{1_{\left\{S_{k}>s, X_{k}=j\right\}}, 0<s \leq t, 1 \leq k \leq n, j \in E, j \neq i\right\} .
$$

Portanto

$$
\begin{aligned}
A_{i}(t) & =\frac{2}{\theta^{2}} \int_{s_{i-1}}^{t}\left(s-\eta_{i}\right) d s \\
& =\left(\frac{t-\eta_{i}}{\theta}\right)^{2}
\end{aligned}
$$

$\operatorname{com} t>\eta_{i}, \quad t_{i-1} \leq t<t_{i}, \quad t_{0}=0$.

Assim

$$
\exp \left[-A_{i}(s)\right]=\bar{F}\left(s_{i} \mid s_{1}, s_{2}, \ldots s_{i-1}\right)
$$




\section{Capítulo 3}

\section{Redundância ativa através da transformação do compensador}

\subsection{Introdução}

Mantivemos neste Capítulo as mesmas condições iniciais do Capítulo 2, no que diz respeito aos tempos de vida dos componentes e dos spares. A principal diferença entre os dois capítulos está na maneira de modelar o problema. Enquanto no Capítulo 2 (e também no Capítulo 1) a redundância ativa foi caracterizada por uma transformação do processo pontual, agora é através da transformação dos compensadores.

Na primeira seção do Capítulo fizemos a modelagem matemática do problema e a caracterização da redundância ativa através dos compensadores, em seguida comparamos a redundância ativa com a redundância standby minimal (veja Bueno (2005a) para mais detalhes sobre este tipo especial de redundância standby). Em seguida estabelecemos condições sob a quais respondemos ao problema da alocação ótima sob duas hipótese.

Primeiro utilizamos o conceito de função $R R_{2}$ e o Teorema de Kwieciński e 
Szekli (1991), e obtivemos também um exemplo construtivo no qual a transformação $2-\exp \left[-A_{i}(t)\right]$ é $R R_{2}$. Estas primeiras seções foram objeto da publicação (in Press) de Bueno e Carmo (2006).

Por último, utilizamos as medidas de probabilidade $P_{i}$ e $P_{j}$, determinadas pela derivada de Radon Nikodym, correspondentes à redundância ativa dos componentes $S_{i}$ e $S_{j}$ respectivamente. 


\subsection{Transformação do compensador}

Como na Seção 2.2, assumimos a hipótese A e dispomos de $n$ spares com tempos de vida $T_{i}$ disponíveis para fazer uma redundância nos tempos de vida $S_{i}, 1 \leq i \leq n$, respectivamente.

A redundância sobre o tempo de vida $S_{i}$ resulta no tempo de vida $S_{i} \vee T_{i}$, com função distribuição

$$
P\left(S_{i} \vee T_{i} \leq t\right)=1-P\left(S_{i} \vee T_{i}>t\right)
$$

Nas condições da Hipótese A do Capítulo 2, teremos

$$
P\left(S_{i} \vee T_{i}>t\right)=2 P\left(S_{i}>t\right)-P\left(S_{i}>t\right)^{2}=2 \bar{F}_{i}(t)-\bar{F}_{i}(t)^{2} .
$$

No caso da independência entre os componentes, no conjunto $\left\{t<S_{i}\right\}$

$$
A_{i}(t)=-\ln P\left(S_{i}>t \mid \Im_{t}\right)
$$

é o $\mathbb{F}$-processo compensador do componente $i$. Desde que

$$
P\left(S_{i} \vee T_{i}>t \mid \Im_{t}\right)=\exp \left[-A_{i}(t)\right]\left(2-\exp \left[-A_{i}(t)\right]\right)
$$

podemos escrever que no conjunto $\left\{t<S_{i} \vee S\right\}$,

$$
-\ln P\left(S_{i} \vee T_{i}>t \mid \Im_{t}\right)=A_{i}(t)-\ln \left(2-\exp \left[-A_{i}(t)\right]\right),
$$

é o $\mathbb{F}$-processo compensador do componente $i$.

Note que estamos considerando

$$
\Im_{t}=\sigma\left\{1_{\left\{S_{i} \leq s\right\}}, s \leq t, 1 \leq i \leq n\right\} .
$$


No caso de componentes dependentes, queremos propor uma transformação do $\mathbb{F}$ processo compensador que represente a operação de redundância ativa, e que preserve a intuição acima. Note que

$$
\begin{aligned}
A_{i}(t)-\ln \left(2-\exp \left[-A_{i}(t)\right]\right) & =\int_{0}^{t} d A_{(s)}-\int_{0}^{t}\left(\frac{\exp \left[-A_{i}(s)\right]}{2-\exp \left[-A_{i}(s)\right]}\right) d A_{i}(s) \\
& =\int_{0}^{t}\left(\frac{2-2 \exp \left[-A_{i}(s)\right]}{2-\exp \left[-A_{i}(s)\right]} d A_{i}(s)\right.
\end{aligned}
$$

Para caracterizar a redundundância ativa, no caso de componentes dependentes, propomos a seguinte transformação:

$$
B_{j}(t)=\int_{0}^{t} \alpha_{j}(s) d A_{j}(s)
$$

onde

$$
\alpha_{i}(s)=\frac{2-2 \exp \left[-A_{i}(s)\right]}{2-\exp \left[-A_{i}(s)\right]}
$$

e $\alpha_{j}(s)=1$ para $j \neq i$.

Tecnicamente procuramos uma medida de probabilidade $Q$, tal que, sob $Q, B_{j}(t)$ seja o $\mathbb{F}$-processo compensador de $N_{j}(t)=1_{\left\{S_{j} \leq t\right\}}$, com respeito a esta nova medida de probabilidade. Isto segue dos Teoremas 3.1 e 3.2 a seguir.

\section{Teorema 3.1:}

O processo $L_{i}(t)$ definido por

$$
L_{i}(t)=\left(\frac{2-2 \exp \left[-A_{i}\left(S_{i}\right)\right]}{2-\exp \left[-A_{i}\left(S_{i}\right)\right]}\right)^{N_{i}(t)}\left(2-\exp \left[-A_{i}(t)\right]\right),
$$

é um $\mathbb{F}$-martingal local.

\section{Prova}


Considere o $\mathbb{F}$-tempo de parada definido por

$$
U_{n}=\inf \left\{t \geq 0: A_{i}(t) \geq n \text { or } B_{i}(t) \geq n\right\}
$$

Claramente tem-se $U_{n} \uparrow \infty$ quando $n \uparrow \infty$.

É suficiente provar que o processo

$$
L_{i}^{n}(t)=\left(\frac{2-2 \exp \left[-A_{i}\left(S_{i}\right)\right]}{2-\exp \left[-A_{i}\left(S_{i}\right)\right]}\right)^{N_{i}\left(t \wedge U_{n}\right)}\left(2-\exp \left[-A_{i}\left(t \wedge U_{n}\right)\right]\right)
$$

é um $\mathbb{F}$-martingal limitado. Assim o processo

$$
L_{i}(t)=\sum_{n=0}^{\infty} 1_{\left\{U_{n} \leq t \leq U_{n+1}\right\}} L_{i}^{n}(t)
$$

é um $\mathbb{F}$-martingal local.

Para todo $\mathbb{F}$-tempo de parada $U \leq U_{n}$, podemos escrever

$$
\begin{gathered}
L^{n}(U)=1-\int_{0}^{U} \exp \left[\int_{0}^{s} \frac{\exp \left[-A_{i}(u)\right]}{2-\exp \left[-A_{i}(u)\right]} d A_{i}(u)\right] \\
.\left(\frac{\exp \left[-A_{i}(s)\right]}{2-\exp \left[-A_{i}(s)\right]}\right) d\left[N_{i}(s)-A_{i}(s)\right]
\end{gathered}
$$

visto que se $U<S_{i}$, teremos $N_{i}(U)=0$ e

$$
\begin{aligned}
1+\int_{0}^{U} \exp \left[\int_{0}^{s}\right. & \left.\frac{\exp \left[-A_{i}(u)\right]}{2-\exp \left[-A_{i}(u)\right]} d A_{i}(u)\right]\left(\frac{\exp \left[-A_{i}(s)\right]}{2-\exp \left[-A_{i}(s)\right]}\right) d A_{i}(s) \\
& =\exp \left[\int_{0}^{U}\left(\frac{\exp \left[-A_{i}(u)\right]}{2-\exp \left[-A_{i}(u)\right]}\right) d A_{i}(u)\right] \\
& =2-\exp \left[-A_{i}(U)\right] \\
& =L^{n}(U) .
\end{aligned}
$$


Se $U \geq S_{i}$

$$
\begin{aligned}
1- & \int_{0}^{U} \exp \left[\int_{0}^{s} \frac{\exp \left[-A_{i}(u)\right]}{2-\exp \left[-A_{i}(u)\right]} d A_{i}(u)\right] \\
& .\left(\frac{\exp \left[-A_{i}(s)\right]}{2-\exp \left[-A_{i}(s)\right]}\right) d\left[N_{i}(s)-A_{i}(s)\right] \\
= & \exp \left[\int_{0}^{S_{i}}\left(\frac{\exp \left[-A_{i}(u)\right]}{2-\exp \left[-A_{i}(u)\right]}\right) d A_{i}(u)\right] \\
& .\left(\frac{2-2 \exp \left[-A_{i}\left(S_{i}\right)\right]}{2-\exp \left[-A_{i}\left(S_{i}\right)\right]}\right) \\
= & 2-2 \exp \left[-A_{i}\left(S_{i}\right)\right] \\
= & L^{n}(U) .
\end{aligned}
$$

Como o integrando

$$
\exp \left[\int_{0}^{s} \frac{\exp \left[-A_{i}(u)\right]}{2-\exp \left[-A_{i}(u)\right]} d A_{i}(u)\right]\left(\frac{\exp \left[-A_{i}(s)\right]}{2-\exp \left[-A_{i}(s)\right]}\right)
$$

é um $\mathbb{F}$-processo previsível e $N_{i}(s)-A_{i}(s)$ é um $\mathbb{F}$-martingal, $L_{i}^{n}(t)$ é um $\mathbb{F}$-martingal ( Bremaud (1982),T6, Cap. 1) e

$$
E\left[L_{i}^{n}(t)\right]=1 .
$$

Teorema 3.2: Sob a medida de probabilidade $Q_{i}$ definida pela derivada de Radon Nikodym

$$
\frac{d Q_{i}}{d P}=L_{i}(\infty)
$$

$B_{j}(t)$ é o $\mathbb{F}$-processo compensador de $N_{j}(t)$.

\section{Prova}


Do Teorema 3.1, $L_{i}(t)$, é um $\mathbb{F}$-martingal com $E\left[L_{i}(t)\right]=1$ e portanto $L_{i}(t)$ pode se considerado como uma função densidade. Denotaremos $Q_{i}$ a medida de probabilidade que é definida pela derivada de Radon Nikodym

$$
\frac{d Q_{i}}{d P}=L_{i}(\infty)
$$

Consideremos a sub $\sigma$-algebra de parada $\mathbb{F}^{n}=\left(\Im_{t \wedge U_{n}}\right)_{t \geq 0}$.

Para todo $\mathbb{F}$-tempo de parada $U \leq U_{n}$ e para $j=i$,

$$
\begin{aligned}
E_{Q_{i}}\left[B_{i}(U)\right] & =E\left[L_{i}(U) B_{i}(U)\right] \\
& =E\left[\int_{0}^{U} L_{i}(s) d B_{i}(s)\right] \\
& =E\left[\int_{0}^{U}\left(2-\exp \left[-A_{i}(s)\right]\right)\left(\frac{2-2 \exp \left[-A_{i}(s)\right]}{2-\exp \left[-A_{i}(s)\right]}\right) d A_{i}(s)\right] \\
& =E\left[\int_{0}^{U}\left(2-2 \exp \left[-A_{i}(s)\right]\right) d N_{i}(s)\right] \\
& =E\left[1_{\left\{S_{i} \leq U\right\}}\left(2-2 \exp \left[-A_{i}\left(S_{i}\right)\right]\right)\right] \\
& =E_{Q_{i}}\left[N_{i}(U)\right] .
\end{aligned}
$$

A segunda igualdade acima segue da Fórmula Integral de Dellacherie A.6.

Agora para $j \neq i$, temos

$$
\begin{aligned}
E_{Q_{i}}\left[B_{j}(U)\right] & =E\left[L_{i}(U) A_{j}(U)\right] \\
& =E\left[\int_{0}^{U} L_{i}(s) d A_{j}(s)\right] \\
& =E\left[\int_{0}^{U} L_{i}\left(s^{-}\right) d A_{j}(s)\right] \\
& =E\left[\int_{0}^{U} L_{i}\left(s^{-}\right) d N_{j}(s)\right] \\
& =E\left[1_{\left\{S_{j} \leq U\right\}} L_{i}\left(S_{j}\right)\right] \\
& =E_{Q_{i}}\left[N_{j}(U)\right]
\end{aligned}
$$


já que $A_{j}(s)$ é um processo natural crescente e $L_{i}\left(S_{j}^{-}\right)=L_{i}\left(S_{j}\right)$ onde $L\left(S_{j}^{-}\right)$é o limite lateral pela esquerda de $L_{i}\left(S_{j}\right)$. 


\subsection{Comparação entre redundância ativa e redundância standby minimal}

Bueno (2005a) definiu um tipo particular de redundância standby, denominada redundância standby minimal, definida a seguir

Definição 3.3: Uma redundâcia standby minimal de um componente com tempo de vida $S_{i}$ é a soma $S_{i}+S$, onde $: \mathrm{S}$, o tempo de vida do spare, é tal que

$$
P\left(S>\mid S_{i}=s\right)=P\left(S_{i}>t+s \mid S_{i}>s\right) .
$$

Utilizando esta definição ele caracterizou a redundância standby minimal através da transformação do compensador dada por:

$$
C_{j}(t)=\int_{0}^{t} \alpha_{j}(s) d A_{j}(s)
$$

onde $\quad \alpha_{i}(s)=\frac{A_{i}(s)}{1+A_{i}(s)} \quad$ e $\quad \alpha_{j}(s)=1 \quad$ para $j \neq i$.

Se decidirmos fazer uma operação de redundância num componente específico, devemos responder qual tipo de redundância, a standby minimal ou a ativa, que deveriamos usar para otimizar estocasticamente o tempo de vida do sistema. Se os componentes são independentes é fácil ver que o tempo de vida devido à operação de redundância standby minimal $\left(S_{i}+S\right)$ é estocásticamente maior que o tempo de vida $S_{i} \vee S$, produzido pela operação de redundância ativa onde $S$ é independente e identicamente distribuído a $S_{i}$.

No caso de dependência, desde que

$$
2-\exp [x] \leq x+1
$$

teremos que a transformação correspondente à redundância ativa

$$
A_{i}(t)-\ln \left(2-\exp \left[-A_{i}(t)\right]\right)
$$


é maior que a correspondente à da redundância standby minimal

$$
A_{i}(t)-\ln \left(1+A_{i}(t)\right)
$$

Do Corolário 1.7, o $\mathbb{F}$-processo compensador de um sistema $k-d e-n: F$ é

$$
A(t)=\sum_{i=1}^{n}\left[A_{i}(t \wedge \tau)-A_{i}(Y)\right]^{+}
$$

e como a função $f(x)=\frac{1+x}{2-\exp [x]}$ é crescente em $x$, temos

$$
\begin{aligned}
C_{i}(t)-C_{i}(Y) & =A_{i}(t)-\ln \left(1+A_{i}(t)\right)-A_{i}(Y)+\ln \left(1+A_{i}(Y)\right) \\
& \leq A_{i}(t)-\ln \left(2-\exp \left[-A_{i}(t)\right]\right)-A_{i}(Y)+\ln \left(2-\exp \left[-A_{i}(Y)\right]\right) \\
& =B_{i}(t)-B_{i}(Y),
\end{aligned}
$$

Assim consideramos que uma redundância ativa produz um tempo de vida inferior ao de uma redundância standby minimal, no sentido de processo de risco de falha do componente $i$ sob uma redundância ativa ser maior que o processo de risco de falha do componente $i$ sob uma redundância standby minimal.

Portanto, se decidirmos fazer uma operação de redundância num componente específico, por exemplo no componente mais fraco de um sistema $k-d e-n: F$, deveriamos usar a redundância standby minimal. 


\subsection{Sistema $k-d e-n: F$ e função $R R_{2}$}

Como nas seções precedentes, denotamos o tempo de vida de um sistema $k-$ de $-n: F$ por $\tau_{k: F}(\mathrm{~S})=S_{(k)}$, onde $\mathbf{S}=\left(S_{1}, \ldots, S_{n}\right)$ é o vetor dos tempos de vida dos componentes do sistema, e o tempo de vida resultante da operação de redundância ativa sobre o componente $i$ por $\tau_{k: F}^{i}=\tau_{k: F}\left(S_{1}, \ldots, S_{i-1}, S_{i} \vee T_{i}, S_{i+1}, \ldots, S_{n}\right)$. O processo de contagem $N^{i}(t)=1_{\left\{\tau_{k: F}^{i} \leq t\right\}}$ conta a falha desse sistema, e $A^{i}(t), \quad 1 \leq i \leq n$ é o seu correspondente $\mathbb{F}$-processo compensador.

Usaremos o resultado seguinte de Kwieciński e Szekli (1991).

Teorema 3.4: (Kwieciński and Szekli (1991))

Considere dois processos pontuais $N$ e $M$ com correspondentes processos compensadores

$$
\begin{aligned}
& A_{n}(t)=A_{n}\left(t \mid t_{0}, t_{1}, \ldots, t_{n-1}\right) \text { sobre }\left(T_{n-1}, T_{n}\right] \\
& B_{n}(t)=B_{n}\left(t \mid s_{0}, s_{1}, \ldots, s_{n-1}\right) \text { sobre }\left(S_{n-1}, S_{n}\right]
\end{aligned}
$$

os quais são contínuos em $t$. Se $A_{n}(t) \leq B_{n}(t)$ para todo $t$ com $s_{0}, s_{1}, \ldots, s_{n-1}$ e $t_{0}, t_{1}, \ldots, t_{n-1}$, tal que $s_{i} \leq t_{i}, 0 \leq i \leq n-1$, então existe uma medida de probabilidade $Q$ tal que

$$
E_{Q}[\psi(N(t))] \leq E_{Q}[\psi(M(t))]
$$

para toda função $\psi$, real, contínua à direita com limite pela esquerda, e decrescente. Que é equivalente a dizer $N \leq^{s t} M$, sob $Q$.

A fim de compararmos os compensadores do sistema correspondente às $n$ opções de alocação, utilizaremos a definição de funções Reversas de Ordem dois, $R R_{2}$ (Reverse Rule of Order 2), da Teoria de Positividade Total, (Karlin (1968). Tais funções 
são usadas para caracterizar as famílias com a propriedade de razão de verossimelhança decrescente( Kotz e Johnson (Ed) (1985)).

\section{Definição 3.5:}

Uma função real e positiva $K(x, y)$, definida para $-\infty<x<\infty$, e $-\infty<y<\infty$ é reversa de ordem dois $\left(R R_{2}\right)$ se, e somente se

$$
K\left(x_{1}, y_{2}\right) K\left(x_{2}, y_{1}\right) \geq K\left(x_{1}, y_{1}\right) K\left(x_{2}, y_{2}\right)
$$

para todo $-\infty<x_{1}<x_{2}<\infty, \infty<y_{1}<y_{2}<\infty$.

Neste ponto é importante relembrar o Teorema 1.5 do Capítulo 1, que estabelece que devemos comparar os processos compensadores dos componentes somente após os níveis críticos dos respectivos componentes.

\section{Teorema 3.6:}

Se a transformação $K(j, t)=2-\exp \left[-A_{j}(t)\right], \quad 1 \leq j \leq n, \quad t \in[0, \infty)$ é $R R_{2}$, então existe uma medida de probabilidade $Q$, tal que, $\operatorname{sob} Q$,

$$
N^{1}(t) \leq^{s t} N^{2}(t) \leq^{s t} \ldots \leq^{s t} N^{n}(t)
$$

\section{Prova}

Segue dos Teoremas 3.1 e 3.2 que a redundância ativa caracterizada pela transformação do compensador $A_{i}(t)$ do componente $i$ é dada por

$$
\begin{aligned}
B_{i}(t) & =\int_{0}^{t}\left(\frac{2-2 \exp \left[-A_{i}(s)\right]}{2-\exp \left[-A_{i}(s)\right]}\right) d A_{i}(s) \\
& =A_{i}(t)-\ln \left(2-\exp \left[-A_{i}(t)\right]\right) .
\end{aligned}
$$

A partir do Corolário 1.7, devemos comparar o valor da esperança dos compen- 
sadores da forma

$$
\begin{aligned}
A^{i}(t)= & \sum_{j=1}^{i-1}\left[A_{j}(t)-A_{j}(Y)\right] \\
& +A_{i}(t)-\ln \left(2-\exp \left[-A_{i}(t)\right]\right)-A_{i}(Y)+\ln \left(2-\exp \left[-A_{i}(Y)\right]\right) \\
& \left.+\sum_{j=i+1}^{n}\left[A_{j}(t)-A_{j}(Y)\right]\right\},
\end{aligned}
$$

com $1 \leq i, j \leq n$.

Sem perda de generalidade, é suficiente provar para $i=1$ e $j=2$.

Da hipótese, $2-\exp \left[-A_{i}(t)\right]$ é $R R_{2}$, ou seja, para $s \leq t$,

$$
2-\exp \left[-A_{1}(s)\right] .2-\exp \left[-A_{2}(t)\right] \leq 2-\exp \left[-A_{1}(t)\right] .2-\exp \left[-A_{2}(s)\right]
$$

logo, tomando $s=Y$, teremos

$$
\left(\frac{2-\exp \left[-A_{1}(t)\right]}{2-\exp \left[-A_{1}(Y)\right]}\right) \geq\left(\frac{2-\exp \left[-A_{2}(t)\right]}{2-\exp \left[-A_{2}(Y)\right]}\right) .
$$

Assim

$$
-\ln \left(\frac{2-\exp \left[-A_{1}(t)\right]}{2-\exp \left[-A_{1}(Y)\right]}\right) \leq-\ln \left(\frac{2-\exp \left[-A_{2}(t)\right]}{2-\exp \left[-A_{2}(Y)\right]}\right)
$$

e então

$$
\begin{aligned}
A^{1}(t)= & A_{1}(t)-\ln \left(2-\exp \left[-A_{1}(t)\right]\right)-A_{1}(Y) \\
& +\ln \left(2-\exp \left[-A_{1}(Y)\right]\right)+A_{2}(t)-A_{2}(Y) \\
& \left.+\sum_{i=3}^{n}\left[A_{i}(t)-A_{i}(Y)\right]\right\} \\
\leq & A_{1}(t)-A_{1}(Y)+A_{2}(t)-\ln \left(2-\exp \left[-A_{2}(t)\right]\right) \\
& -A_{2}(Y)+\ln \left(2-\exp \left[-A_{2}(Y)\right]\right) \\
& \left.+\sum_{i=3}^{n}\left[A_{i}(t)-A_{i}(Y)\right]\right\} \\
= & A^{2}(t) .
\end{aligned}
$$


O resultado segue do Teorema 3.4

Como $A_{i}(0)=0$ para todo $i$, se $2-\exp \left[-A_{i}(t)\right]$ é $R R_{2}$, teremos $A_{i}(t) \geq A_{j}(t)$ para todo $i \leq j$. Portanto interpretamos que o componente $i$ é mais fraco que o componente $j$, ou seja, o risco de falha do componente $i$ é maior que o do componente $j$.

Sob o Teorema 3.6, otimizamos a confiabilidade do sistema $k-d e-n: F$ escolhendo o componente mais fraco para alocarmos o componente redundante. Como não fizemos referência a qualquer relação entre os componentes do sistema, o resultado vale para componentes dependentes.

No caso de componentes estocasticamente independentes, e valendo a hipótese de que $2-\exp \left[-A_{i}(t)\right]$ é $R R_{2}$ podemos provar que se $A_{i}(t) \geq A_{j}(t)$ então $S_{i} \leq^{s t} S_{j}$ para todo $i \leq j$, e temos o corolário a seguir.

\section{Corolário 3.7:}

Sejam $\left\{S_{1}, \ldots, S_{n}\right\}$ os tempos de vida, que são estocasticamente independentes, dos componentes de um sistema $k-d e-n: F$. Se $2-\exp \left[-A_{i}(t)\right]$ é $R R_{2}$, então $S_{1} \leq^{s t} S_{2} \leq^{s t} \ldots \leq^{s t} S_{n}$ e $\tau_{k: F}^{1} \geq^{s t} \tau_{k: F}^{2} \geq^{s t} \ldots \geq^{s t} \tau_{k: F}^{n} \quad$ para $\quad k=1, \ldots, n$.

\section{Exemplo 3.8:}

Sejam $\left\{S_{1}, \ldots, S_{n}\right\}$ tempos de vida estocasticamente independentes de um sistema $k-d e-n: F$. Se o i-ésimo tempo de vida de um componente desse sistema tem distribuição Gamma de parâmetros $\lambda$ e $i, \lambda>0$ e $i=1, \ldots, n$, então $S_{1} \leq^{\text {st }} S_{2} \leq^{\text {st }}$ $\ldots \leq^{s t} S_{n}$, e escolhemos o primeiro componente, $i=1$, para fazer a redundância. Neste caso o tempo de vida do componente "1", aumenta de $S_{1}$ para $S_{1} \vee S$, onde $S$ 
é o tempo de vida do componente redundante que é independente e identicamente distribuido como $S_{1}$. 


\subsection{Exemplo construtivo onde $2-\exp \left[-A_{i}(t)\right]$ é $R R_{2}$}

Propomos encontrar tempos de vida $S_{i}^{*}, \quad i=1, \ldots, n$, com $\mathbb{F}$-processo compensador $A_{i}^{*}(t)$ de $1_{\left\{S_{i}^{*} \leq t\right\}}$ tal que

$$
2-\exp \left[-A_{j}^{*}(t)\right]
$$

tenha a propriedade $R R_{2}$.

Consideramos como em Arjas (1981a), um tempo de vida $S_{i}$ (ou sua distribuição), que tem taxa de falha crescente relativo a $\Im_{t}$. Porém, como $S_{i}$ é $\mathbb{F}$-mensurável, temos

$$
P\left(S_{i}>t \mid \Im_{t}\right)=1_{\left\{S_{i}>t\right\}}
$$

o que não é apropriado para nosso propósito.

Consideraremos então um tempo de vida que tem taxa de falha crescente relativo a $\Im_{t}^{i}$, onde

$$
\Im_{t}^{i}=\sigma\left\{1_{\left\{S_{j}>s\right\}}, s \leq t, j=1, \ldots, n, j \neq i\right\}
$$

Assim, $S_{i}$ é $I F R \mid \Im_{t}^{i}$, isto é,

$$
P\left(\left(S_{i}-t\right)^{+}>s \mid \Im_{t}^{i}\right) \quad \downarrow t, \quad P-q . c .
$$

Claramente teremos também

$$
\bar{F}_{i}(t)=P\left(S_{i}>t \mid \Im_{t}^{i}\right) \quad \downarrow t, \quad P-q . c .
$$

Seja $M_{i}(t)$ uma versão que é contínua à direita com limite à esquerda do processo

$$
M_{i}(t)=E\left[1_{\left\{S_{i} \leq t\right\}} \mid \Im_{t}^{i}\right]
$$


Segue que $M_{i}(t)$ é um $\Im_{t}^{i}$-submartingal com $\Im_{t}^{i}$-compensador $C_{i}(t)$. De Arjas (1981b), se $S_{i}$ é $\left(I F R \mid \Im_{t}^{i}\right), C_{i}(t)$ é $P-q . c$. convexo sobre $\left(0, S_{i}\right]$. Agora, como

$$
M_{i}(t)-C_{i}(t)
$$

é um $\Im_{t}^{i}$-martingal, para $s<t$, teremos

$$
E\left[P:\left(S_{i} \leq t \mid \Im_{t}^{i}\right)-P\left(S_{i} \leq s \mid \Im_{s}^{i}\right) \mid \Im_{s}^{i}\right]=E\left[C_{i}(t)-C_{i}(s) \mid \Im_{s}^{i}\right]
$$

e portanto, segue do Teorema da Convergência Monótona que

$$
\lim _{s \rightarrow t} E\left[P\left(S_{i} \leq t \mid \Im_{t}^{i}\right)-P\left(S_{i} \leq s \mid \Im_{s}^{i}\right) \mid \Im_{s}^{i}\right]=0
$$

isto é,

$$
\lim _{s \rightarrow t} \int_{B}\left[P\left(S_{i} \leq t \mid \Im_{t}^{i}\right)-P\left(S_{i} \leq s \mid \Im_{s}^{i}\right) \mid \Im_{s}^{i}\right] d P=0
$$

para todo $B \in \Im_{i}^{i}$.

Como

$$
P\left(S_{i}>t \mid \Im_{t}^{i}\right) \downarrow t
$$

$P\left(S_{i} \leq t \mid \Im_{t}^{i}\right)$ é contínuo à esquerda e portanto $\Im_{t}^{i}$-previsível. Segue que

$$
C_{i}(t)=P\left(S_{i} \leq t \mid \Im_{t}^{i}\right)
$$

Consideremos agora o $\Im_{t}$-compensador de $N_{i}(t)=1_{\left\{S_{i} \leq t\right\}}$, onde

$$
\Im_{t}=\Im_{t}^{i} \vee \sigma\left\{1_{\left\{S_{i}>s\right\}}, \mid s \leq t\right\}
$$

Arjas e Yashin (1988) prova que o $\Im_{t^{-}}$compensador de $N_{i}(t), A_{i}(t)$ é dado por

$$
\begin{aligned}
A_{i}(t) & =\int_{0}^{t} \frac{1_{\left\{S_{i}>s\right\}} d C_{i}(s)}{\bar{F}_{i}(s-)} \\
& =-\ln \left(\bar{F}_{i}\left(t \wedge S_{i}\right)\right.
\end{aligned}
$$


Procuramos um $\Im_{t}$-compensador de $N_{i}(t), A_{i}^{*}(t)$, uma transformação de $A_{i}(t)$ tal que

$$
\left.2-\exp \left[-A_{j}^{*}(t)\right]\right)
$$

tenha a propriedade $R R_{2}$. Como $S_{i}$ é $\left(I F R \mid \Im_{t}^{i}\right)$, podemos convenientemente escolher $\bar{F}_{i}(t)$ como uma função $P F_{2}$, ( uma função $T P_{2}$ sob shift) tal que $2-\exp \left[-A_{j}^{*}(t)\right]$ tenha a propriedade $R R_{2}$. Propomos a transformação do compensador

$$
A_{i}^{*}(t)=\int_{0}^{t} \frac{1}{2 \exp \left[-A_{i}(s)\right]-1} d A_{i}(s) .
$$

num domínio apropriado.

Seja agora

$$
\begin{aligned}
L_{i}(t)= & \left(\frac{1}{2 \exp \left[-A_{i}(s)\right]-1}\right)^{N_{i}(t)} \exp \left[A_{i}(t)-A_{i}^{*}(t)\right] \\
= & 1-\int_{0}^{t} \exp \left[-\int_{0}^{s} \frac{\left(2 \exp \left[-A_{i}(u)\right]-2\right)}{\left(2 \exp \left[-A_{i}(u)\right]-1\right)} d A_{i}(u)\right] \\
& \cdot\left[\frac{\left(2 \exp \left[-A_{i}(s)\right]-2\right)}{\left(2 \exp \left[-A_{i}(s)\right]-1\right)}\right] d\left[N_{i}(s)-A_{i}(s)\right] .
\end{aligned}
$$

Como $N_{i}(s)-A_{i}(s)$ é um $\Im_{t}$-martingal e o integrando é $\Im_{t}$-previsível, $L_{i}(t)$ é um $\Im_{t}$-martingal local. Supomos que $L_{i}(t)$ é uniformemente integrável.

Contudo,

$$
E\left[L_{i}(t)\right]=1
$$

assim $L_{i}(t)$ pode ser condiderado como uma função densidade e podemos definir uma medida $Q_{i}$ pela derivada de Radon Nikodym

$$
\frac{d Q_{i}}{d P}=L_{i}\left(S_{i}\right)
$$

Portanto, aplicando o Teorema de Girsanov (Bremaud (1981)) teremos que $A_{i}^{*}(t)$ é o $\Im_{t}$-compensador de $N_{i}(t)$ sob a medida $Q_{i}$. 
Segue que

$$
\begin{aligned}
2 \operatorname{cxp}\left(\left[A_{j}^{*}(t)\right]\right) & =2-\exp \left[\int_{0}^{t} \frac{1}{2 \exp \left[-A_{i}(s)\right]-1} d A_{i}(s)\right] \\
& =\exp \left[A_{i}(t)\right] \\
& =\frac{1}{\bar{F}_{i}(t)}
\end{aligned}
$$

o qual tem a propriedade $R R_{2}$.

Definimos o tempo de vida do componente $S_{i}^{*}$ por:

$$
Q\left(S_{i}^{*}>t \mid \Im_{t}\right)=\exp \left[-A_{i}^{*}(t)\right]
$$

com $1 \leq i \leq n$.

Para dar um exemplo prático, considere novamente os tempos de vida ordenados com função de sobrevivência condicionada como no exemplo 2.5:

$$
\bar{F}\left(t_{i} \mid t_{1}, t_{2}, \ldots t_{i-1}\right)=\exp \left[-\left(\frac{t_{i}-\eta_{i}}{\theta}\right)^{\beta}+\left(\frac{t_{i-1}-\eta_{i}}{\theta}\right)^{\beta}\right]
$$

para $\eta_{i} \vee t_{i-1}<t_{i}$, onde $t_{i}$ são as observações ordenados e com função densidade:

$$
\begin{aligned}
f\left(t_{1}, t_{2}, \ldots, t_{n}\right)= & \prod_{i=1}^{n} f\left(t_{i} \mid t_{1}, t_{2}, \ldots t_{i-1}\right) \\
= & \left(\frac{\beta}{\theta}\right)\left(\frac{t_{1}-\eta_{1}}{\theta}\right)^{\beta-1} \exp \left[\left(\frac{t_{1}-\eta_{1}}{\theta}\right)^{\beta}\right] \\
& \cdot \prod_{i=2}^{n}\left(\frac{\beta}{\theta}\right)\left(\frac{t_{i}-\eta_{i}}{\theta}\right)^{\beta-1} \exp \left[-\left(\frac{t_{i}-\eta_{i}}{\theta}\right)^{\beta}+\left(\frac{t_{i-1}-\eta_{i}}{\theta}\right)^{\beta}\right] .
\end{aligned}
$$

Segue que

$$
\begin{aligned}
d A_{i}\left(t \mid t_{1}, t_{2}, \ldots t_{i-1}\right) & =\frac{f\left(t \mid t_{1}, t_{2}, \ldots t_{i-1}\right)}{\bar{F}\left(\mid t_{1}, t_{2}, \ldots t_{i-1}\right)} \\
& =\left(\frac{\beta}{\theta}\right)\left(\frac{t-\eta_{i}}{\theta}\right)^{\beta-1}
\end{aligned}
$$

com $t_{i-1} \leq t<t_{i}, \quad t_{0}=0$. 
Em particular podemos tomar $\beta=2$, e neste caso $S_{i}$ é apropriadamente uma distribuição $\left(I F R \mid \Im_{t}^{i}\right)$ tal que $\bar{F}\left(t_{i} \mid t_{1}, t_{2}, \ldots t_{i-1}\right)$ é $T P_{2}$ (sob shift) sobre $t_{i}$ e $\eta_{i}$.

Portanto

$$
\begin{aligned}
A_{i}(t) & =\frac{2}{\theta^{2}} \int_{\eta_{i}}^{t}\left(s-\eta_{i}\right) d s \\
& =\left(\frac{t-\eta_{i}}{\theta}\right)^{2},
\end{aligned}
$$

com $t>\eta_{i,}, \quad t_{i-1} \leq t<t_{i}, \quad t_{0}=0$

e

$$
\begin{aligned}
A_{i}^{*}(t) & =\int_{0}^{t} \frac{1}{2 \exp \left[-A_{i}(s)\right]-1} d A_{i}(s) \\
& =-\ln \left(2-\exp \left[\left(\frac{t-\eta_{i}}{\theta}\right)^{2}\right]\right)
\end{aligned}
$$

sobre $\eta_{i}+0,83 \theta>t \geq \eta_{i}, \quad t_{i-1} \leq t<t_{i}, t_{0}=0, \quad i=1, \ldots, n$.

Segue que podemos definir o tempo de vida do componente $S_{i}^{*}$ por :

$$
Q\left(S_{i}^{*}>t \mid \Im_{t}\right)=2-\exp \left[\left(\frac{t-\eta_{i}}{\theta}\right)^{2}\right]
$$

sobre

$$
\eta_{i}+0,83 \theta>t \geq \eta_{i}, \quad i=1, \ldots, n .
$$




\subsection{Sistema $k-d e-n: F$ e as medidas $P_{i}$ e $P_{j}$}

Com relação ao componente $i$, seja $A_{i}(t)$ o $\left(P, \Im_{t}\right)$-processo compensador do processo $1_{\left\{S_{i} \leq t\right\}}, i \in E$, que é não decrescente, $P$-q.c. único, previsível com $A_{i}(0)=$ 0 . Admitimos que $1_{\left\{S_{i} \leq t\right\}}$ tenha intensidade $a_{i}(t)$, de forma que

$$
A_{i}(t)=\int_{0}^{t} 1_{\left\{S_{i}>s\right\}} a_{i}(s) d s .
$$

Portanto, para $t \geq S_{i}$

$$
\begin{aligned}
A_{i}(t) & =\int_{0}^{S_{i}} 1_{\left\{S_{i}>s\right\}} a_{i}(s) d s+\int_{S_{i}}^{t} 1_{\left\{S_{i}>s\right\}} a_{i}(s) d s \\
& =\int_{0}^{S_{i}} 1_{\left\{S_{i}>s\right\}} a_{i}(s) d s \\
& =A_{i}\left(S_{i}\right) .
\end{aligned}
$$

que é uma variável aleatória com distribuição exponencial de parâmetro 1. Prova-se que as variáveis $A_{1}\left(S_{1}\right), A_{2}\left(S_{2}\right), \ldots, A_{n}\left(S_{n}\right)$, são i.i.d., independentes e identicamente distribuídas (Norros (1986)).

Considere novamente o processo $L_{i}(t)$,

$$
L_{i}(t)=\left(\frac{2-2 \exp \left[-A_{i}\left(S_{i}\right)\right]}{2-\exp \left[-A_{i}\left(S_{i}\right)\right]}\right)^{N_{i}(t)}\left(2-\exp \left[-A_{i}(t)\right]\right)
$$

$L_{i}(t)$ é um $\left(P_{i}, \Im_{t}\right)$-martingal local. Desde que é limitado, é um $\left(P_{i}, \Im_{t}\right)$-martingal.

Note que

$$
L^{i}=\lim _{t \uparrow \infty} L^{i}(t)=L^{i}=2-\exp \left[-A_{i}\left(S_{i}\right)\right]=L^{i}\left(S_{i}\right)
$$

Assim definimos $P_{i}$ como a derivada de Radon Nikodim

$$
\frac{d P_{i}}{d P}=L_{i}\left(S_{i}\right)
$$

Segue que 


$$
\begin{aligned}
P_{i}(\Omega) & =\int_{\Omega} L^{i}\left(S_{i}\right) d P \\
& =\mathbb{E}\left[L^{i}\left(S_{i}\right)\right] \\
& =\mathbb{E}\left[L^{i}(0)\right] \\
& =\mathbb{E}[1]=1
\end{aligned}
$$

Portando, $L_{i}\left(S_{i}\right)$ pode ser considerado como uma função densidade sob a medida de probabilidade $P_{i}$ e

$$
\mathbb{E}_{P_{i}}\left[A_{i}(t)\right]=\mathbb{E}\left[A_{i}(t) \cdot L^{i}\left(S_{i}\right)\right]
$$

Os $B_{m}(t) \mathbb{F}$-compensadores de $1_{\left\{S_{i} \leq t\right\}}$ sob a nova medida $P_{i}$, representando a redundância ativa sobre do componente $i$, conforme Seção 3.2 é

$$
B_{m}(t)=\int_{0}^{t} \alpha_{m}(s) d A_{m}(s)
$$

onde

$$
\alpha_{i}(s)=\frac{2-2 \exp \left[-A_{i}(s)\right]}{2-\exp \left[-A_{i}(s)\right]}
$$

e $\alpha_{m}(s)=1$ para $m \neq i$, ou seja,

$$
B_{i}(t)=A_{i}(t)-\ln \left[2-\exp \left(-A_{i}(t)\right)\right]
$$

Para um $k-d e-n: F$, com nível crítico $Y$, teremos

$$
\begin{aligned}
A^{i}(t) & =\sum_{m=1}^{n}\left[B_{m}(t \wedge \tau)-B_{m}(Y)\right]^{+} \\
& =1_{\{Y \leq t \wedge \tau\}} \sum_{m=1}^{n}\left[B_{m}(t \wedge \tau)-B_{m}(Y)\right] .
\end{aligned}
$$


Logo,

$$
\begin{aligned}
\mathbb{E}_{P_{i}}\left[A^{i}(t)\right] & =\mathbb{E}_{P_{i}}\left\{1_{\{Y \leq t \wedge \tau\}} \sum_{m=1}^{n}\left[B_{m}(t \wedge \tau)-B_{m}(Y)\right]\right\} \\
& =\mathbb{E}_{P_{i}}\left\{\mathbb{E}_{P_{i}}\left\{1_{\{Y \leq t \wedge \tau\}} \sum_{m=1}^{n}\left[B_{m}(t \wedge \tau)-B_{m}(Y)\right] \mid \Im_{Y}\right\}\right\} \\
& \stackrel{*}{=} \mathbb{E}_{P_{i}}\left\{1_{\{Y \leq t \wedge \tau\}} \mathbb{E}_{P_{i}}\left\{\sum_{m=1}^{n}\left[B_{m}(t \wedge \tau)-B_{m}(Y)\right] \mid \Im_{Y}\right\}\right\} \\
& =\mathbb{E}_{P_{i}}\left\{1_{\{Y \leq t \wedge \tau\}} \sum_{m=1}^{n} \mathbb{E}_{P_{i}}\left\{\left[B_{m}(t \wedge \tau)-B_{m}(Y)\right] \mid \Im_{Y}\right\}\right\} \\
& =\mathbb{E}_{P_{i}}\left\{1_{\{Y \leq t \wedge \tau\}} \sum_{m=1}^{n} \mathbb{E}_{P_{i}}\left\{1_{\left\{S_{m} \leq t \wedge \tau\right\}}-M_{m}(t \wedge \tau)-1_{\left\{S_{m} \leq Y\right\}}+M_{m}(Y) \mid \Im_{Y}\right\}\right\} \\
& =\mathbb{E}_{P_{i}}\left\{1_{\{Y \leq t \wedge \tau\}} \sum_{m=1}^{n} \mathbb{E}_{P_{i}}\left[1_{\left\{S_{m} \leq t \wedge \tau\right\}}-1_{\left\{S_{m} \leq Y\right\}} \mid \Im_{Y}\right]\right\} \\
& =\mathbb{E}_{P_{i}}\left\{\sum_{m=1}^{n} \mathbb{E}_{P_{i}}\left[1_{\{Y \leq t \wedge \tau\}} \cdot\left(1_{\left\{S_{m} \leq t \wedge \tau\right\}}-1_{\left\{S_{m} \leq Y\right\}}\right) \mid \Im_{Y}\right]\right\} \\
& =\mathbb{E}_{P_{i}}\left\{\mathbb{E}_{P_{i}}\left\{\sum_{m=1}^{n}\left[1_{\{Y \leq t \wedge \tau\}} \cdot\left(1_{\left\{S_{m} \leq t \wedge \tau\right\}}-1_{\left\{S_{m} \leq Y\right\}}\right)\right] \mid \Im_{Y}\right\}\right\} \\
& =\mathbb{E}_{P_{i}}\left\{1_{\{Y \leq t \wedge \tau\}} \cdot \sum_{m=1}^{n}\left(1_{\left\{S_{m} \leq t \wedge \tau\right\}}-1_{\left\{S_{m} \leq Y\right\}}\right)\right\} \\
& \mathbb{E}_{\left.L^{i} .1_{\{Y \leq t \wedge \tau\}} \cdot \sum_{m=1}^{n}\left(1_{\left\{S_{m} \leq t \wedge \tau\right\}}-1_{\left\{S_{m} \leq Y\right\}}\right)\right\} .}, \\
& \\
&
\end{aligned}
$$

Em (*), $1_{\{Y \leq t \wedge \tau\}}$ é $\Im_{Y}$-mensurável, veja Dellacherie e Meyer (1980) (Teorema 6, Cap. 1).

Análogamente, obtém-se que

$$
\mathbb{E}_{P_{j}}\left[A^{j}(t)\right]=\mathbb{E}\left\{L^{j} \cdot 1_{\{Y \leq t \wedge \tau\}} \cdot \sum_{m=1}^{n}\left(1_{\left\{S_{m} \leq t \wedge \tau\right\}}-1_{\left\{S_{m} \leq Y\right\}}\right)\right\}
$$

Assim

$$
\begin{aligned}
\mathbb{E}_{P_{i}}\left[A^{i}(t)\right]-\mathbb{E}_{P_{j}}\left[A^{j}(t)\right] & =\mathbb{E}\left\{\left[L^{i}-L^{j}\right] \cdot 1_{\{Y \leq t \wedge \tau\}} \cdot \sum_{m=1}^{n}\left(1_{\left\{S_{m} \leq t \wedge \tau\right\}}-1_{\left\{S_{m} \leq Y\right\}}\right)\right\} \\
& =\mathbb{E}\left\{\left[L^{i}-L^{j}\right] \cdot \sum_{m=1}^{n}\left[1_{\left\{S_{m} \leq t \wedge \tau\right\}}-1_{\left\{S_{m} \leq Y\right\}}\right]^{+}\right\} .
\end{aligned}
$$


Portanto a diferença $\mathbb{E}_{P_{i}}\left[A^{i}(t)\right]-\mathbb{E}_{P_{j}}\left[A^{j}(t)\right]$ tem o mesmo sinal de $L^{i}-L^{j}$. Logo temos o seguinte teorema:

\section{Teorema 3.9:}

Sob as condições acima, se

$$
A_{1}(t) \geq A_{2}(t) \geq \ldots \geq A_{n}(t), \quad P-q . c .
$$

então

$$
\mathbb{E}_{P_{1}}\left[A^{1}(t)\right] \geq \mathbb{E}_{P_{2}}\left[A^{2}(t)\right] \geq \ldots \geq \mathbb{E}_{P_{n}}\left[A^{n}(t)\right]
$$

\section{Prova}

Observe inicialmente que $A_{i}\left(S_{i}\right) \geq A_{j}\left(S_{j}\right)$, pois

sob $\left\{S_{i} \leq S_{j}\right\}$

$$
A_{i}\left(S_{i}\right) \geq A_{j}\left(S_{i}\right)=A_{j}\left(S_{j}\right)
$$

por outro lado, sob $\left\{S_{i} \leq S_{j}\right\}$,

$$
A_{i}\left(S_{i}\right)=A_{j}\left(S_{i}\right) \geq A_{j}\left(S_{j}\right)
$$

Assim

$$
\begin{aligned}
L^{i}-L^{j} & =\left(2-2 \exp \left[-A_{i}\left(S_{i}\right)\right]\right)-\left(2-2 \exp \left[-A_{j}\left(S_{j}\right)\right]\right) \\
& =2 \cdot\left[A_{i}\left(S_{i}\right)-A_{j}\left(S_{j}\right)\right] \\
& \geq 0 .
\end{aligned}
$$

Desde que $A_{i}(t) \geq A_{j}(t)$ implica em $S_{i} \leq^{\text {st }} S_{j}, i \leq j, i, j \in E$, e que $P_{i}$ é uma medida correspondendo á redundância ativa do componente $i$, deve-se fazer a redundância ativa no componente "mais forte". 


\section{Apêndice A}

\section{Alguns resultados utilizados nos Capítulos}

Teorema A.1: (Decomposição de Doob-Meyer)

Seja $X=\left(X_{t}\right)_{t \geq 0}$ um processo aleatório contínuo à direita e adaptado a filtragem $\left(\Omega, \Im, F^{n}, \mathbb{P}\right)$. Então $X$ é um submartingal uniformemente integrável se, e somente se, tem a decomposição

$$
X=A+M
$$

onde $A=\left(A_{t}\right)_{t \geq 0}$ é um processo previsível, contínuo à direita, não-decrescente, e integrável, com $\quad A_{0}=0 \quad$ e $\quad M=\left(M_{t}\right)_{t \geq 0}$ é um martingal uniformemente integrável. A decomposição é única a menos de indistingüibilidade. 
Proposição A.2: (Integração por partes)

Sejam $a$ e $b$ funções cadlag sobre $\mathbb{R}_{+}$, de variação limitada. Então, para cada $t \in \mathbb{R}_{+}$

$$
a(t) \cdot b(t)=\int_{o}^{t} a\left(s^{-}\right) a(d s)+\int_{o}^{t} b(s) a(d s)+\sum_{s \leq t} \Delta a(s) \cdot \Delta b(t),
$$

onde $a\left(s^{-}\right)$é o limite pela esquerda em $s$ e $\Delta a(s)=a(s)-a\left(s^{-}\right)$.

Lema A.3: (Dellacherie e Meyer (1980))

Seja $(\Omega, \Im, \mathbf{F}, P)$ um espaço de probabilidade filtrado e $T$ um $\mathbf{F}$-tempo de parada.

(i) Se o processo $V=\left(V_{t}\right), V_{t}=I(T \leq t)$, tem uma representação semimartingal

$$
V_{t}=\int_{0}^{t} I(T \geq s) A_{s} d s+M_{t}, t \in \mathbb{R}_{+}
$$

então $T$ é um tempo de parada totalmente inacessivel e o martigal $M$ é quadrado integrável de média zero.

(ii) Se $T$ é um tempo de parada totalmente inacessivel então o processo $V=$ $\left(V_{t}\right), V_{t}=I(T \leq t)$, tem uma única (P-q.c.) decomposição $V=A+M$, onde $M$ é um martingal U.I. e $A$ é contínuo (P-q.c., compensador previsível).

Teorema A.4: (Boland)

Sejam $S, S_{1}, S_{2}, \ldots, S_{n}$ variáveis aleatórias independentes, representando os tempos de vida de um spare e dos componentes de um sistema $k-d e-n: G$, respectivamente. Se

$$
S_{1} \leq^{s t} S_{2} \leq^{s t} \ldots \leq^{s t} S_{n}
$$

então

$$
\tau_{k: n}^{1} \geq^{s t} \tau_{k: n}^{2} \geq^{s t} \ldots \geq^{s t} \tau_{k: n}^{n}
$$

onde $\tau_{k: n}^{i}$ representa o tempo de vida do sistema com redundância ativa sobre o componente $i, 1 \leq i \leq n$. 
Teorema A.5: Bueno (2005b)

Sejam $\tau, S_{1}, S_{2}, \ldots, S_{n}$, os tempos de vida de um sistema coerente e de seus componentes, respectivamente. Considere $S_{i}^{*}=\left[\left(S_{i}-Y_{i}\right)^{+} \mid \Im_{Y_{i}}\right], 1 \leq i \leq n$, onde $Y_{i}$ é o nível crítico do componente $i$. Sob a $\sigma$-álgebra $\Im_{Y+t}$, se

$$
\tau^{*}=\min _{\left\{i: Y_{i}<\infty\right\}} S_{i}^{*}
$$

então

$$
\tau=\tau^{*},
$$

$P$-q.c., onde $\Im_{Y+t}=\left\{A \in \Im_{\infty}: A \cap\{Y+t \leq s\} \in \Im_{s}, s \geq 0\right\}$.

\section{Prova:}

Observe inicialmente que

$$
1_{\left\{S_{i}^{*} \leq t\right\}}=\mathbb{E}\left[N_{i}\left(Y_{i}+t\right)-N_{i}\left(Y_{i}\right) \mid \Im_{Y_{i}}\right]=\mathbb{E}\left[1_{\left\{Y_{i}<T_{i} \leq Y_{i}+t\right\}} \mid \Im_{Y_{i}}\right]
$$

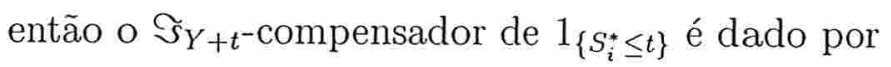

$$
C_{i}(t)=\mathbb{E}\left[A_{i}\left(Y_{i}+t\right)-A_{i}\left(Y_{i}\right) \mid \Im_{Y_{i}}\right]
$$

pois, $N_{i}(t)-A_{i}(t)$ é um $\Im_{t}$-martingal uniformemente integrável, e assim aplicando o Teorema da Amostragem Opcional, para $s \leq t$, temos

$$
\mathbb{E}\left[N_{i}\left(Y_{i}+t\right)-A_{i}\left(Y_{i}+t\right) \mid \Im_{Y_{i}+s}\right]=N_{i}\left(Y_{i}+s\right)-A_{i}\left(Y_{i}+s\right)
$$

Então

$$
\begin{gathered}
\mathbb{E}\left\{\mathbb{E}\left[N_{i}\left(Y_{i}+t\right)-N_{i}\left(Y_{i}\right) \mid \Im_{Y_{i}}\right]-\mathbb{E}\left[A_{i}\left(Y_{i}+t\right)-A_{i}\left(Y_{i}\right) \mid \Im_{Y_{i}}\right] \mid \Im_{Y_{i}+s}\right\}= \\
\mathbb{E}\left\{\mathbb{E}\left[N_{i}\left(Y_{i}+t\right)-A_{i}\left(Y_{i}+t\right) \mid \Im_{Y_{i}}\right] \mid \Im_{Y_{i}+s}\right\}-\mathbb{E}\left\{\mathbb{E}\left[N_{i}\left(Y_{i}\right)-A_{i}\left(Y_{i}\right) \mid \Im_{Y_{i}}\right] \mid \Im_{Y_{i}+s}\right\}= \\
\mathbb{E}\left[N_{i}\left(Y_{i}+s\right)-A_{i}\left(Y_{i}+s\right) \mid \Im_{Y_{i}}\right]-\mathbb{E}\left[N_{i}\left(Y_{i}\right)-A_{i}\left(Y_{i}\right) \mid \Im_{Y_{i}}\right]
\end{gathered}
$$




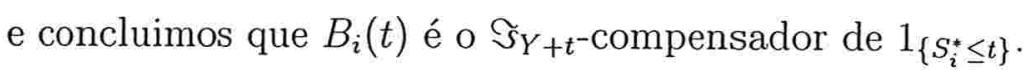

Por outro lado $\tau \in \Im_{t} \mathrm{e}$

$$
\{\tau=s\} \Leftrightarrow\left\{\tau \in \bigcap_{1 \leq m<\infty}\left[s, s+\frac{1}{m}\right)\right\} \Leftrightarrow\left\{s \in \bigcap_{1 \leq m<\infty}\left(\tau-\frac{1}{m}, \tau\right]\right\}
$$

é um conjunto previsível. Logo $1_{\{\tau=s\}}$ é um $\Im_{t}$-processo previsível.

Agora $\{\tau=s\}=\bigcup_{i \in \Gamma_{\phi}(s)}\left\{T_{i}=s\right\}$ e segue que $w \in\{\tau=s\}$ se, e somente se, $w \in\left\{T_{i}=s\right\}$ para algum $i \in \Gamma_{\phi}(s)$. Contudo $\left\{i \in \Gamma_{\phi}(s)\right\} \cap\left\{T_{i}=s\right\}$ é equivalente a $\left\{Y_{i}<T_{i}=\tau=s\right\}$ e $\left\{Y_{i}<T_{i}\right\} \in \Im_{Y_{i}} \subset \Im_{Y_{i}+s}$. Assim $\{\tau=s\} \in \Im_{Y_{i}+s}$ e portanto $1_{\{\tau=s\}}$ é um $\Im_{Y_{i}+t}$-processo previsível.

Dessa forma, se $N(t)=1_{\{\tau \leq t\}}$ e $N^{*}(t)=1_{\left\{\tau^{*} \leq t\right\}}$ teremos

$$
\begin{gathered}
1=P(\tau=\tau)=\mathbb{E}\left[\int_{0}^{\infty} 1_{\{\tau=s\}} d N(s)\right] \stackrel{a}{=} \mathbb{E}\left[\int_{0}^{\infty} 1_{\{\tau=s\}} d A(s)\right] \stackrel{b}{=} \\
\mathbb{E}\left[\int_{0}^{\infty} 1_{\{\tau=s\}} d A^{*}(s)\right] \stackrel{c}{=} \mathbb{E}\left[\int_{0}^{\infty} 1_{\{\tau=s\}} d N^{*}(s)\right]=\mathbb{E}\left[1_{\left\{\tau=\tau^{*}\right\}}\right]=P\left(\tau=\tau^{*}\right) .
\end{gathered}
$$

A igualdade (a) segue da $\Im_{t}$-previsibilidade de $\tau,(b)$ do conceito de esperança condicional e $(c)$ deve-se ao fato de que $\tau$ também é $\Im_{Y_{i}+s}$-tempo de parada previsível.

Teorema A.6: (Fórmula Integral de Dellacherie)

Sejam $A_{t}$ um processo crescente adaptado a $\Im_{t}$, e $M_{t}$ um $\left(P, \Im_{t}\right)$-martingal, contínuo a direita e uniformemente integrável. Então, para todo $\Im_{t}$-tempo de parada $T$

$$
\mathbb{E}\left[\int_{0}^{T} M_{t} d A_{t}\right]=\mathbb{E}\left[M_{T} A_{T}\right]
$$




\section{Referências Bibliográficas}

[1] E. Arjas: A stochastic process approach to multivariate reliability system: notions based on conditional stochastic order, Mathematics of Operations Research, 6(2) (1981a), 263-276.

[2] E. Arjas: The failure and hazard processes in multivariate reliability systems, Mathematics of Operations Research, 6 (1981b), 551-562.

[3] E. Arjas and A. Yashin: A note on random intensities and conditional survival functions, Journal of Applied Probability. 25 (1988), 630 - 635.

[4] T. Aven e U. Jensen: Stochatic Models in Reliability, Springer-Verlag, New York (1999).

[5] L. J. Bain: Statistical analysis of reliability and life-testing models: theory and methods, Marcel Dekker, New York (1978).

[6] R. E. Barlow and F. Proschan: Statistical Theory of Reliability and Life Testing: Probability models, Hold, Rinehart and Wiston, Silver Spring, MD, (1981).

[7] Boland P., El-Neweihi E., Proschan F. : Stochastic order for redundancy allocations in series and parallel systems, Advanced Applied Probability. 24 (1992), 161-171. 
[8] P. Bremaud: Point Processes and Queues, Springer-Verlag, New York (1982).

[9] V.C. Bueno: Minimal standby redundancy allocation in a. $k-$ mıt $-o f-n: F$ system of dependent components, European Journal of Operational Research. 165 (2005a), $786-793$.

[10] V.C. Bueno: A series representation of a coherent system, RT-MAE-IMEỦniversidade de São Paulo, 20 (2005b).

[11] V. C. Bueno e I. M. Carmo: Active redundancy allocation for a $k-$ out - of $-n$ : F system of dependent components, European Journal of Operational Research, (In Press) (2006).

[12] C. Dellacherie e P.A. Meyer: Probabilities and Potential, B. North-Holland, Amsterdam (1980).

[13] S. Karlin: Total Positivity, Stanford, University Press (1968).

[14] S. Kotz and N. Johnson,(Eds): Encyclopedia of Statistical Sciences. Volume 5. Wiley, New York (1985).

[15] A. Kwieciński and R. Szekli : Compensator conditions for stochastic ordering of point processes, Journal of Applied Probability. 28 (1991), 751-761.

[16] F. C. Meng: More on optimal allocation of components in coherent systems, Journal of Applied Probability. 33 (1996), 548-556.

[17] J. Mi: A unified way of comparing the reliability of coherent systems, IEEE Transactions on Reliability, 52 (2003), 38-43.

[18] I. Norros: A compensator representation of multivariate lifelength distributions, with applications, Scandinavian Journal of Statistics, 13 (1986),99-112. 
[19] Prasad, V.R., Kuo, W. and Oh Kim, K.M. : Optimal allocation of identical, multi functional sapres in a series system, IEEE Transactions on Reliability. 48 (1999), 2, 118-126.

[20] M. Shaked and J. G. Shanthikumar: Stochastic orders and their applications, Academic Press, New York (1994).

[21] Singh," H. and Misra, N. : On redundancy allocation in systems, Journal of Applied Probability. 31 (1994), 1004 - 1014. 